\title{
硼酸酯键在药物传递体系中的应用
}

\author{
王李娟 $b$ 盛显良 ${ }^{a}$ 王 杰 $a$ 张玉辉*, $a$ \\ ( ${ }^{a}$ 内蒙古农业大学理学院 呼和浩特 010018) \\ ${ }^{b}$ 内蒙古农业大学材料科学与艺术设计学院 呼和浩特 010018)
}

\begin{abstract}
摘要 刺激响应性药物载体由于其优异的控释性能, 在生物医药领域引发了广泛的关注并得到了极为快速的发展. 硼 酸酯键因构筑条件简单、生物相容性好以及能够响应生物体内 $\mathrm{pH}$ 、葡萄糖、三磷酸腺苷(ATP)等多种微环境变化的优 势被广泛用于刺激响应性药物载体的构筑. 基于厥酸酯键的药物载体类型有药物-聚合物偶联、聚合物胶束、线性-超 支化聚合物和介孔二氧化硅等，它们既能负载抗癌药物，又能递送胰岛素和基因. 药物通过共价或非共价作用负载到 载体上, 并利用硼酸酯键在不同环境下的形成与断裂实现药物的可控释放. 从药物类型、载体类型、药物与载体的结 合方式以及嗍酸酯键的断裂机制四个方面综述了硼酸酯键在药物传递体系中的应用, 并对其当前面临的主要挑战和未 来的发展趋势进行了总结和展望.
\end{abstract}

关键词 刺激响应; 药物载体; 嗍酸酯键

\section{Application of Boronate Bond in Drug Delivery System}

\author{
Wang, Lijuan ${ }^{b} \quad$ Sheng, Xianliang ${ }^{a} \quad$ Wang, Jie $^{a} \quad$ Zhang, Yuhui ${ }^{*, a}$ \\ ( ${ }^{a}$ College of Science, Inner Mongolia Agricultural University, Hohhot 010018) \\ $\left({ }^{b}\right.$ College of Material Science and Art Design, Inner Mongolia Agricultural University, Hohhot 010018)
}

\begin{abstract}
Because of its excellent controlled release properties, stimulus-responsive drug carriers have attracted extensive attention in the field of biomedicine and have achieved extremely rapid development. Boronate bonds have been widely used as a stimulus-responsive site in the construction of drug carriers because of its simple construction conditions, good biocompatibility, and fast responsiveness to various micro-environmental changes such as $\mathrm{pH}$, glucose and 5'-adenosine triphosphate (ATP) concentration in vivo. The types of drug carriers based on boronate bonds include drug-polymer conjugate, polymer micelles, linear-hyperbranched polymers and mesoporous silica, which could load anticancer drugs and deliver insulin and genes. Besides, drugs could be integrated into carrier by covalent bonding, physical encapsulation, and electrostatic interactions, which could be released at specific sites via endogenous stimuli. The application of boronate bond in drug delivery system is reviewed from four aspects: drug types, carrier types, combination mode of drug and carrier as well as breaking mechanism of boronate bond. The main challenges and future advances in this field are also detailed.
\end{abstract}

Keywords stimulus-responsive; drug carrier; boronate bond

目前, 许多药物在临床应用过程中由于水溶性差及 毒副作用大等缺点未能充分发挥药效. 众所周知, 药物 经静脉注射进入人体后, 需要经过血液循环、在病变部 位富集、在病变组织中渗透、被细胞内吞以及在细胞内 释放五个步骤才能起到治疗作用 ${ }^{[1]}$. 人体组织发生病变 后, 会与正常组织存在诸多差异, 如血管通透性变差、
$\mathrm{pH}$ 下降或血糖浓度升高等, 利用这些特点构筑刺激响 应性药物载体成为改善药物疗效的有效手段. 其中, 具 有亲水性表面的纳米药物载体具有独特的优势 ${ }^{[2]}$ 。一方 面，亲水性表面能够使药物在血液循环过程中逃避免疫 系统的识别而获得长时间循环性能; 另一方面, 病变组 织中血管壁间隙较宽, 结构完整性较差, 淋巴回流缺失,

\footnotetext{
* Corresponding author. E-mail: yh_zhangyh@126.com

Received June 28, 2020; revised August 4, 2020; published online September 15, 2020.

Project supported by the Program for Young Talents of Science and Technology in Universities of Inner Mongolia Autonomous Region (No. NJYT-19-B26), the Doctor Fund of Inner Mongolia Autonomous Region Natural Science Fund (No. 2017BS0206) and the Program of Higher-level Talents Introduction and Scientific Research Start of Inner Mongolia Agricultural University (No. NDGCC2016-21).

内蒙古自治区高等学校 “青年科技英才支持计划” (No. NJYT-19-B26)、内蒙古自治区自然科学基金博士基金(No. 2017BS0206)及内蒙古农业大学高 层次人才引进科研启动(No. NDGCC2016-21)资助项目.
} 
纳米载体可通过增强的渗透滞留(EPR)效应选择性在病 变部位富集.

基于此, 刺激响应性纳米载体因能够实现药物的可 控释放得到了诸多关注 ${ }^{[3-5]}$. 其中, 动态共价键 ${ }^{[6]}$ 作为一 种在适当条件下能够可逆断裂和生成的共价键, 与同样 具有可逆性的超分子非共价键相比, 具有更为优越的稳 固性, 同时具备不可逆共价键所没有的对特殊环境的响 应性，被选为优良的刺激响应位点. 尽管亚胺键、二硫 键及二硒键等动态共价键已被用于药物载体的研究, 但 仍存在诱导条件不温和、会对生物分子造成破坏以及响 应灵敏性差等问题, 对于其在生物体系的应用带来了限 制 $[7-9]$.

近年来, 硼酸因其独特的化学性质被广泛应用于构 筑刺激响应性药物载体的响应位点 ${ }^{[10-11]}$. 首先, 在没有 任何外部辅助条件的情况下, 硼酸与二醇基团能够在水 中迅速发生酯化反应, 生成嗍酸酯键, 且该反应在酸性 条件下可逆. 1959 年, Ewards 等 ${ }^{[12]}$ 发现了硼酸盐的阴离 子结构，阐明了其中的原因: 在 $\mathrm{pH}$ 大于其 $\mathrm{p} K_{\mathrm{a}}$ 的中性或 弱碱性介质中, 嗍酸呈四面体构型的解离状态, 能够与 含 1,2-或 1,3-邻二醇结构的化合物快速结合, 形成硼酸 酯键; 在 $\mathrm{pH}$ 远小于其 $\mathrm{p} K_{\mathrm{a}}$ 的酸性介质中, 嗍酸呈平面三 角形构型的非解离状态, 难以同其它化合物结合. 其次, 研究表明，当同时存在多种含邻二醇结构的化合物时， 呈四面体结构的硼酸会优先与键合常数 $\left(K_{\mathrm{a}}\right)$ 较高的化合 物结合 ${ }^{[13-14]}$. 因此, 硼酸酯键对生物体内的葡萄糖、三 磷酸腺苷(ATP)及过氧化氢 $\left(\mathrm{H}_{2} \mathrm{O}_{2}\right)$ 等物质高度敏感. 再 者, 由于中心嗍原子上存在空的 $\mathrm{p}$ 轨道, 硼酸主要作为 路易斯酸起作用, 能够与 $\mathrm{N}$ 等含孤对电子的原子形成配 位键. 此外, 苯硼酸能与癌细胞表面过表达的唾液酸化 蛋白(SA)特异性结合, 还可以赋予药物载体靶向性 ${ }^{[15]}$.

目前, 已有一些含硼酸的药物进入了临床应用. 如 嗍替佐米和依沙佐米是分别于 2003 年和 2015 年经美国 食品药品监督管理局(FDA)批准的用于多发性骨髓瘤治 疗的蛋白酶体抑制剂. 众所周知, 蛋白酶体会降解癌细 胞中的促调亡蛋白. 嗍替佐米 ${ }^{[16]}$ 和依沙佐米 ${ }^{[17]}$ 则分别 通过其结构中的硼原子与 $26 \mathrm{~S}$ 蛋白酶体活性位点和 $20 \mathrm{~S}$ 蛋白酶体的 $\beta 5$ 亚单位结合来抑制蛋白酶体的活性, 使 得癌细胞中的促调亡蛋白不会被降解, 进而导致细胞周 期停滞和调亡. 又如, 克立硼罗 ${ }^{[18]}$ 是 2016 年经 FDA 批 准的用于轻度至中度特应性皮炎的局部治疗药物. 其结 构中的嗍原子能够与磷酸二酷酶 4 (PDE4)的双金属中 心结合, 导致环状单磷酸腺苷(cAMP)含量升高, 从而抑 制 NF-kB 通路并抑制促炎性介质(如 TNF- $\alpha$ 和各种白介 素)的释放，治疗特应性皮炎.

本文从药物类型、载体类型、药物与载体的结合方
式以及嗍酸酯键的断裂机制四个方面综述了硼酸酯键 在药物递送体系中的应用, 并对其当前面临的主要挑战 和未来的发展趋势进行了总结和展望, 以期为今后的研 究提供新思路.

\section{1 药物类型}

\section{1 抗癌药物}

癌症是当今世界威胁人类生命健康的最大杀手. 根 据世界卫生组织报告, 全球每年新发 1000 万癌症患者, 有 $600 \sim 700$ 万人死亡, 癌症的发病率和死亡率正逐渐 上升 ${ }^{[19]}$. 目前, 化疗是治疗癌症最常用的方法, 但在临 床应用过程中存在水溶性差及毒副作用大等问题, 会对 正常细胞造成不同程度的损害，给患者带来极大痛苦， 甚至造成患者死亡 ${ }^{[20]}$. 构筑响应于肿瘤微环境从而实 现药物定位释放的载体, 能够改善或克服以上问题, 减 轻患者痛苦，提高治疗效果. 硼酸酯键能够响应于肿瘤 组织的酸性 $\mathrm{pH}$ 而被用于构筑抗癌药物载体.

冯俊等 ${ }^{[21}$ 将苯嗍酸修饰到胆固醇上(Chol-PBA), 然 后使其通过硼酸酯键与葡聚糖(Dex)结合，自组装成具 有酸响应性的纳米粒子 Dex/Chol-PBA，其可将药物靶 向输送至溶酶体内(图 1). 他们以阿霉素(DOX)为模型 药物, 测得纳米粒子对 DOX 的负载率为 $8.6 \%$, 包封率 为 $81.7 \%$, 并具有很好的 $\mathrm{pH}$ 响应释放能力. 在 $\mathrm{pH}=7.4$ 的环境中孵育，纳米粒子较稳定，第一阶段只有 $20 \%$ 的 药物释放, $10 \mathrm{~d}$ 后的释放量也不到 $40 \%$, 而在 $\mathrm{pH}=5$ 的 条件下，第一阶段就有 $60 \%$ 的药物快速释放. 将该纳米 粒子在正常血糖浓度(约 $1.0 \mathrm{mg} / \mathrm{L}$ )和含血清 $(\varphi=10 \%)$ 的 条件下静置 $6 \mathrm{~d}$ 后, 粒径和粒度分布均无显著变化, 证 明了其在生理条件下的稳定性. 分别将磷酸缓冲溶液 $(\mathrm{PBS})$ 、负载 DOX 的 Dex/Chol-PBA 和游离的 DOX 注 射到携带肿瘤体积 $150 \mathrm{~mm}^{3}$ 的小鼠体内, $12 \mathrm{~d}$ 后观察到 空白对照组的小鼠肿瘤长到 $1600 \mathrm{~mm}^{3}$, 比初始体积增 大 11 倍; 注射负载 DOX 的纳米粒子组的小鼠肿瘤生长 得到明显抑制, 体重变化不明显, 正常细胞的存活率为 $100 \%$; 注射游离 DOX 组的小鼠肿瘤生长也得到明显抑 制，但体重明显下降，正常细胞的存活率不足 $100 \%$. 由 此可见，使用 Dex/Chol-PBA 负载抗癌药物不仅能够保 留药物的抗癌活性, 还可以降低对正常细胞的毒副作 用.

随后，张云等 ${ }^{[22]}$ 为提高药物负载率，分别使 3,4-二 羟基苯甲醛和 4-甲酰基苯硼酸与对苯二胺反应，制备了 两种前体, Im-Ca 和 Im-Ba(图 2). 然后利用 Im-Ca 和 $\mathrm{Im}-\mathrm{Ba}$ 之间以及 $\mathrm{Im}-\mathrm{Ba}$ 和 DOX 之间形成的硼酸酯键，制 备了一种能够通过共价键作用负载 DOX 的纳米载体 (BNP), 使得载药率达到 $30 \%$. 此外, 为了增加药物在 


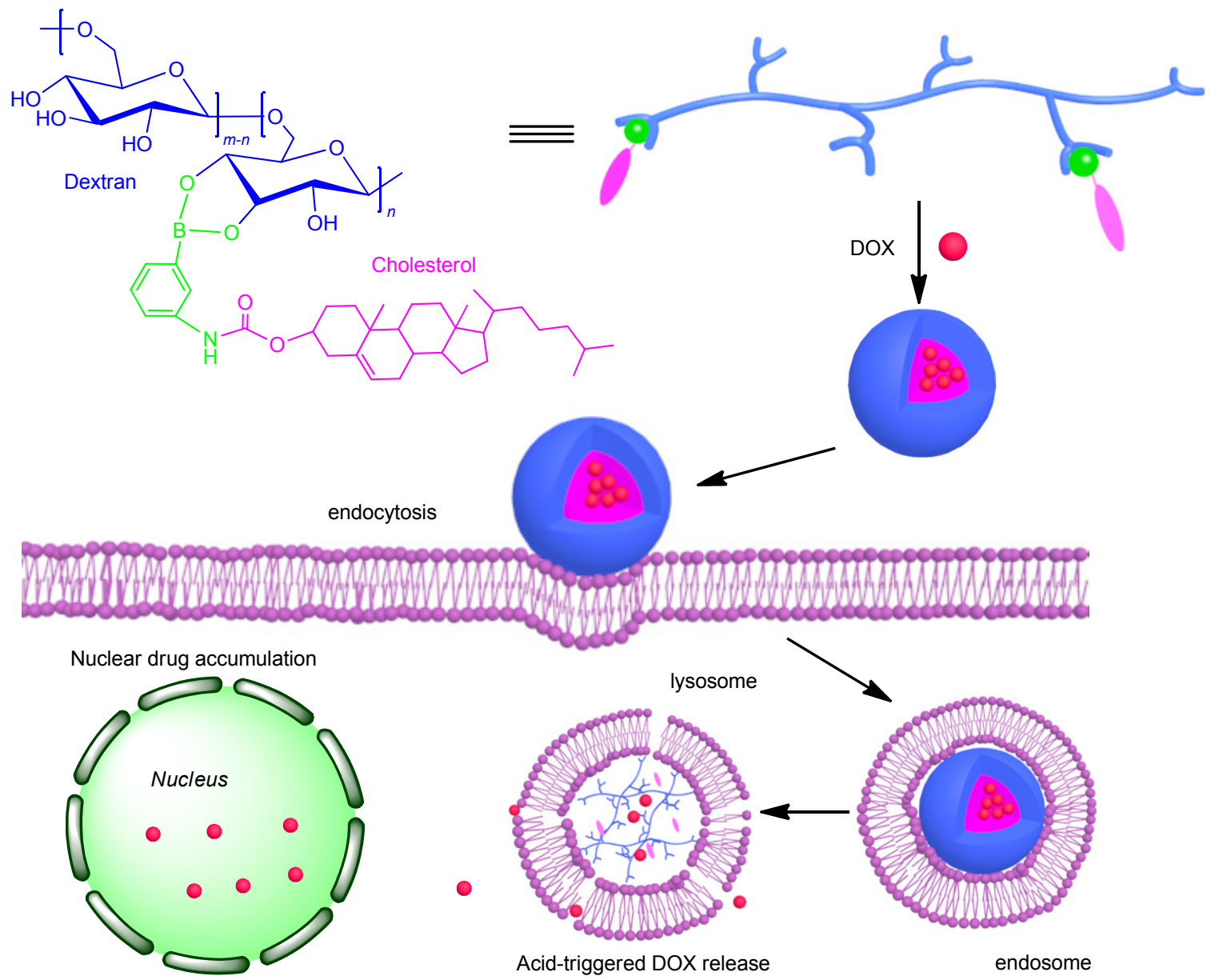

图 1 Dex/Chol-PBA 纳米组装体的形成过程及其用于溶酶体酸度靶向的 $\mathrm{pH}$ 响应的药物递送示意图

Figure 1 Schematic illustration of the formation process of Dex/Chol-PBA nanoassembly and its pH-responsive drug delivery for lysosomal acidity targeting

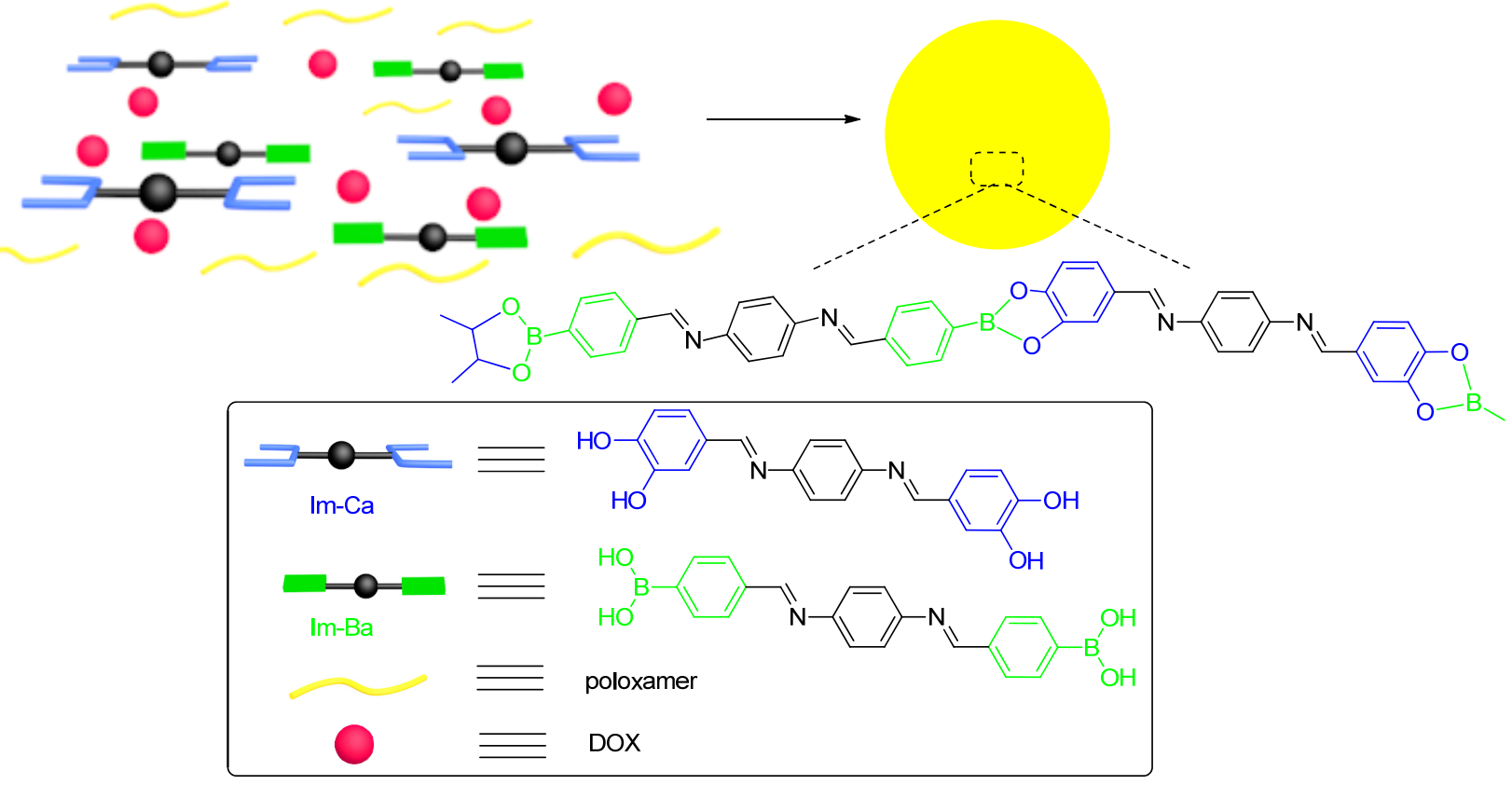

图 $2 \mathrm{DOX} / \mathrm{BNPs}$ 纳米组装体的形成过程示意图

Figure 2 Schematic illustration of the formation process of DOX/BNPs nanoassembly 
血液循环过程中的稳定性, 他们在药物负载过程中加入 泊洛沙姆 188 , 成功地给 DOX/BNP 表面涂覆了一层能 够响应酸性微环境的涂层, 这使得药物在血液循环过程 中实现零释放, 而当纳米颗粒到达肿瘤组织时, 酸性微 环境使得涂层脱落, Im-Ca 和 Im-Ba 之间的硼酸酯键断 开, 促进药物的释放与吸收.

\section{2 胰岛素}

糖尿病是一种慢性内分泌疾病，患者体内胰岛素或 升糖激素分泌紊乱, 会引起代谢性酸中毒及高酮血症等 并发症, 进而危及生命 ${ }^{[23]}$. 近年来, 我国糖尿病发病率 呈现不断上升趋势, 根据资料统计, 糖尿病人数已超过 4000 万, 居世界第 2 位 ${ }^{[24]}$. 因糖尿病难以彻底治愈, 摄 入外源胰岛素是控制病情发展的主要治疗方法. 目前, 胰岛素的给药方式主要有两种, 皮下注射和胰岛素 泵 ${ }^{[25]}$. 皮下注射给药量有限, 多次注射会导致皮下脂肪 萎缩和硬结, 增加患者痛苦. 胰岛素洜虽能解决上述问 题, 但其软针部分需长期埋在皮下, 极易引发感染; 此 外, 作为需要挂在身上的外来物品, 给患者的日常生活 带来诸多不便, 降低了患者的生活质量. 因此, 开发能 够响应于葡萄糖浓度的智能药物递送体系是解决胰岛 素给药难题的关键手段. 硼酸酷键因能被葡萄糖通过竞 争性交换反应诱导解离而备受研究者的青崃.

柴志华等 ${ }^{[26]}$ 利用聚(3-甲基丙烯酰胺基苯硼酸) (PMAPBA) 与葡聚糖 (Dex) 之间的硼酸-二醇络合作用 (图 3), 制备了一种平均粒径为 $100 \mathrm{~nm}$ 左右的球形纳米 粒子(PMAPBA-Dex), 用于胰岛素的递送. 通过 UV-Vis 测得该纳米粒子对胰岛素的负载率为 $22 \%$, 包封率为 $85 \%$, 相对较高. 将负载胰岛素的 PMAPBA-Dex 分别在 没有葡萄糖以及含 1 和 $3 \mathrm{mg} / \mathrm{mL}$ 葡萄糖的溶液中睬育 7 $\mathrm{h}$ 后, 测得胰岛素的释放量分别为 $42 \%, 60 \%$ 和 $95 \%$. 说 明在 $3 \mathrm{mg} / \mathrm{mL}$ 葡萄糖存在下药物几乎从纳米颗粒中完 全释放出来. 将NIH3T3 细胞在含 PMAPBA-Dex 的培养 基中孵育 $48 \mathrm{~h}$ 后, 细胞相对存活率超过 $80 \%$, 说明 PMAPBA-Dex 的细胞毒性较低. 圆二色光谱表明胰岛
素的结构在负载过程中未发生改变. 因此, PMAPBA-Dex 可作为一种有效的胰岛素输送载体，提高治疗 效果, 减少不良反应.

刘育等 ${ }^{[27]}$ 利用苯硼酸修饰的 $\beta$-环糊精和金刚烷修 饰的聚乙烯亚胺之间的主客体作用构筑了一种具有匍 萄糖响应性的同时表达和传输胰岛素的超分子聚多糖 纳米簇. 电镜实验显示其呈粒径为 $183 \mathrm{~nm}$ 的球形纳米 粒子, zeta 电位为 $45.8 \mathrm{mV}$, 有利于细胞的摄取和 DNA 的负载. 由 FITC-胰岛素的紫外标准曲线计算载体对胰 岛素的包封率和负载率分别为 $84.4 \%$ 和 $8.4 \%$. 肤岛素的 释放实验表明, 载体在葡萄糖浓度为 $5 \mathrm{mg} / \mathrm{mL}$ 的环境下 的释放速率比没有葡萄糖时高 5.4 倍，证明了其对葡萄 糖的响应性. 此外, 流式细胞仪测得载体的基因表达效 率高达 $32 \%$, 高于商业化转染试剂 $\mathrm{PEI}^{25 \mathrm{k}}(25 \%)$. 酶联免 疫吸附测定表明, HepG2 细胞与载体共同孵育后的胰岛 素分泌水平为 $21.13 \mathrm{mIU} / \mathrm{L}$, 是空白对照组的 2.2 倍. 这 些结果表明, 载体能够提高胰岛素分泌水平, 在治疗糖 尿病方面具有潜在应用价值.

王乐勇等 ${ }^{[28]}$ 利用水溶性柱 [5]芳烃 $\left(\mathrm{WP}_{5}\right)$ 与吡啶硼酸 衍生物 $\left(\mathrm{G}_{1}\right)$ 之间的主客体相互作用, 自组装形成具有葡 萄糖响应性的超分子囊泡, 用于胰岛素传递研究(图 4). 随后，他们又合成了双苯硼酸衍生物 $\left(\mathrm{G}_{2}\right)$, 利用其与水 溶性柱 [5]芳烃之间的主客体相互作用自组装形成超分 子囊泡, 用于胰岛素和葡萄糖氧化酶 (GOx) 负载(图 $5)^{[29]}$. 实验表明, 负载胰岛素和 $\mathrm{GO} x$ 的囊泡在高血糖环 境中, 能够选择性识别葡萄糖进而导致囊泡结构破坏并 释放胰岛素. 此外, 释放出的 $\mathrm{GO} x$ 可氧化葡萄糖生成葡 萄糖酸使体系的 $\mathrm{pH}$ 降低并释放出 $\mathrm{H}_{2} \mathrm{O}_{2}$, 从而进一步加 速药物释放. 小鼠实验进一步证实, 这种多重刺激响应 性超分子诊疗体系可对高血糖环境进行快速响应，并可 有效地调节 I 型糖尿病小鼠的葡萄糖水平，为糖尿病诊 疗提供了新的思路.

顾臻等 ${ }^{[30]}$ 合成了一种带有侧链氨基和苯硼酸(PBA) 基团的聚合物，聚 $[((2$-丙烯酰胺基乙基)氨基甲酸

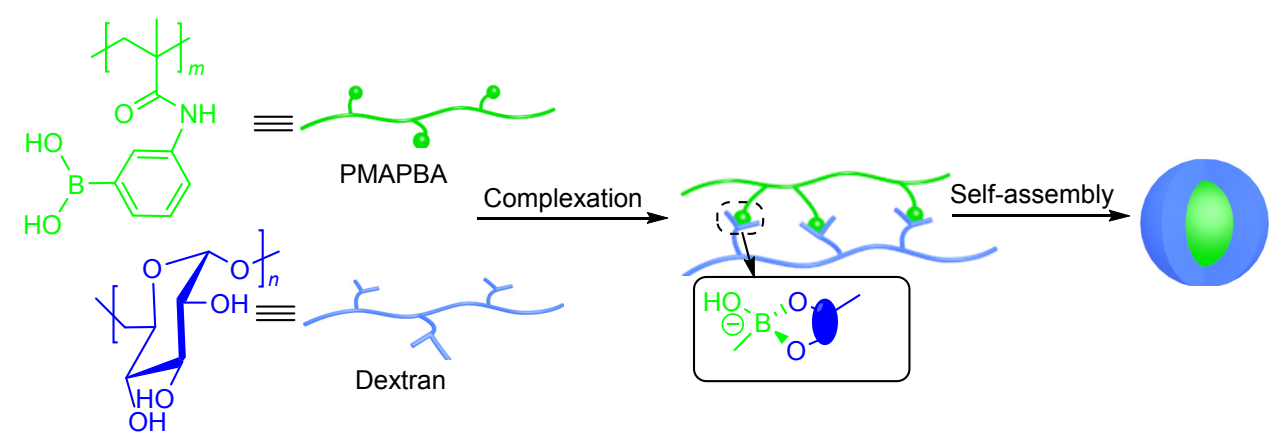

图 3 PMAPBA/Dextran 纳米组装体形成过程的示意图

Figure 3 Schematic illustration of the formation process of PMAPBA/Dextran nanoassembly 

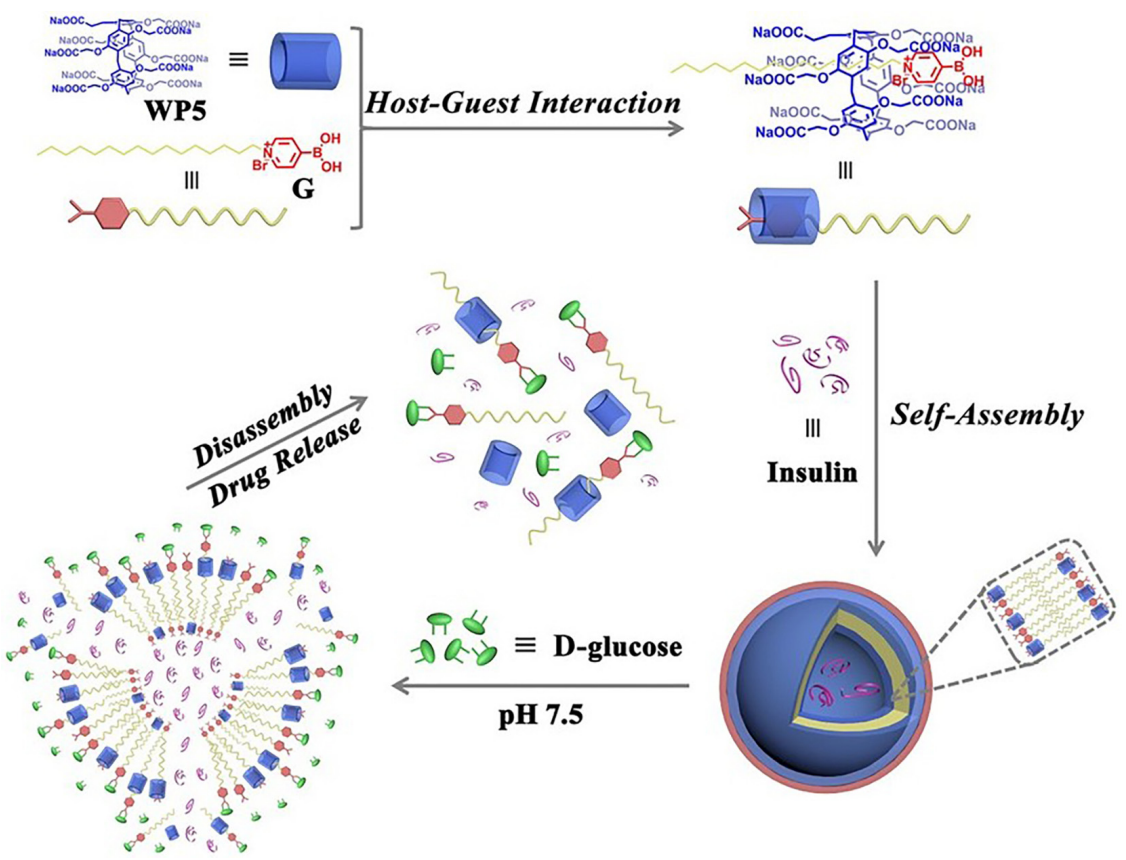

图 4 基于 WP5 $\mathrm{GG}$ 超两亲物的超分子囊泡的形成过程及其葡萄糖响应的胰岛素递送示意图 ${ }^{[28]}$

Figure 4 Schematic illustration of the formation process of supramolecular vesicles based on WP5つG supra-amphiphile and their glucose-responsive insulin delivery ${ }^{[28]}$
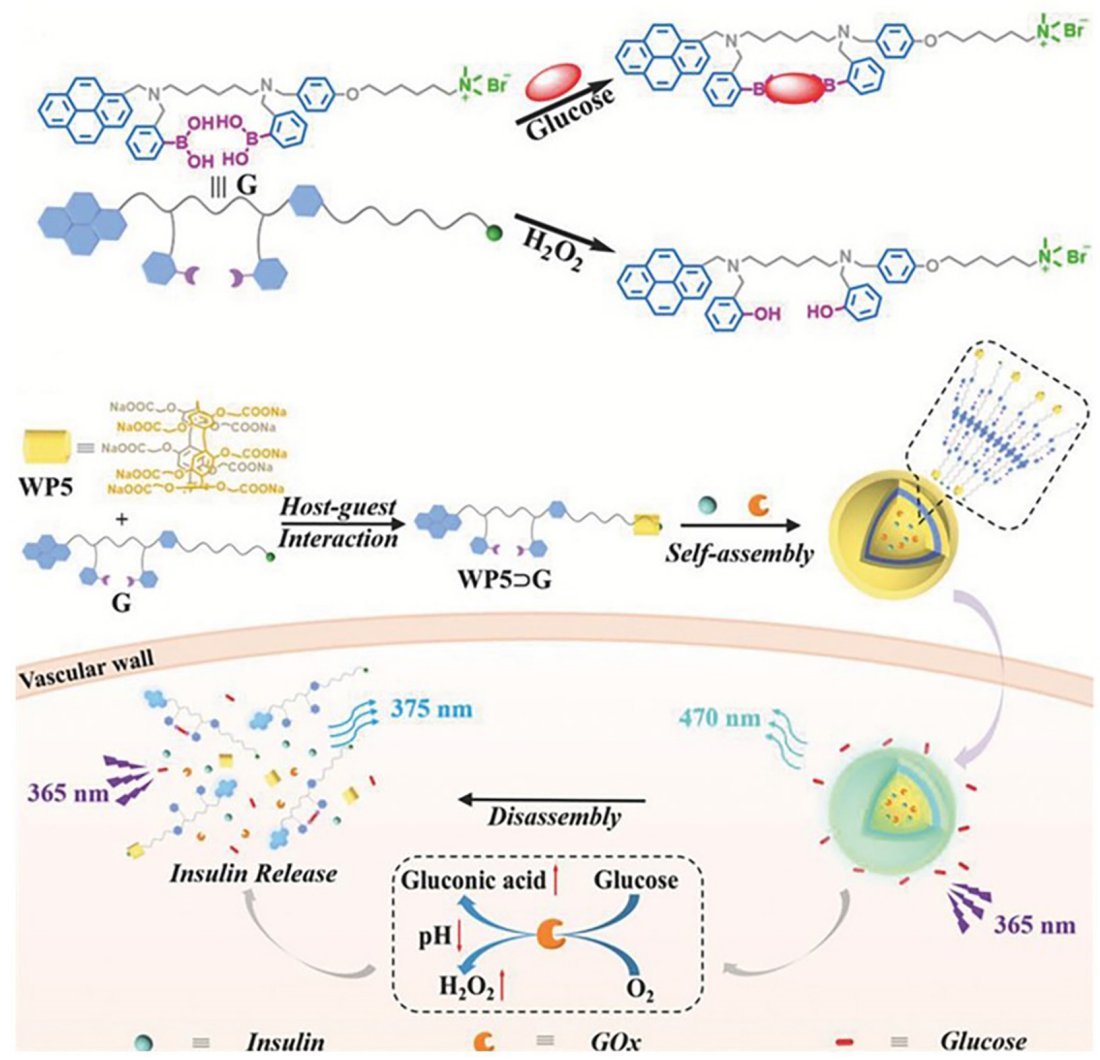

图 5 主体-客体复合物 WP5 $5 \mathrm{G}$ 的超分子囊泡形成过程及其在高糖状态下的胰岛素和 $\mathrm{GO} x$ 递送示意图 ${ }^{[29]}$

Figure 5 Schematic illustration of the formation process of supramolecular vesicles of the host-guest complex WP5つG as well as their insulin and GOx delivery under hyperglycemic state ${ }^{[29]}$

酯 $)_{0.4}$-(4-羧基-3-氟苯基硼酸) 0.6 ( EDAA $_{0.4}-\mathrm{FPBA}_{0.6}$ ). 在 $\mathrm{pH}=7.4$ 的磷酸缓冲溶液中, 该聚合物带正电, 能够通
过静电作用负载带负电的胰岛素，包封率高达 95\%，负 载率高达 $49 \%$. 聚合物中 PBA 与葡萄糖的结合会降低 
正电荷密度, 削弱聚合物对胰岛素的静电吸引。在葡萄 糖浓度较高的条件下, 聚合物的电荷会逆转, 从而促进 肤岛素的快速释放. 同时, 血糖水平恢复正常后, 这种 电荷逆转被抑制, 降低了胰岛素释放速率, 从而降低了 发生低血糖症的风险. 随后, 他们 ${ }^{[31]}$ 又制备了一种响应 于葡萄糖的微针贴剂 (GR-MN), 整个聚合物基质(聚 $(N-$ 乙烯基吡咯烷酮-co-2-丙烯酸(二甲氨基)乙酯-co-3-(丙 烯酸酰胺基)PBA))都充当了葡萄糖响应部分. 实验表 明, GR-MN 贴片突破了普通贴剂载药率不高的瓶颈, 并 显示出与皮肤组织良好的生物相容性, 可在较长时间内 对葡萄糖水平进行葡萄糖响应性调节, 还为胰岛素类似 物等其它药物的刺激响应性透皮递送系统的开发提供 了新思路.

\section{3 基因}

基因治疗 ${ }^{[32]}$ 是指运用载体将治疗基因递送到患者 的细胞中, 通过替代突变基因、敲除异常表达基因或补 充缺失基因，治疗或治愈疾病. 基因治疗主要用于治疗 遗传病、恶性肿瘤及感染性疾病等严重疾病, 对维持人 类生命健康具有重大意义. 目前, 基因载体 ${ }^{[33]}$ 主要有两 大类, 病毒载体和非病毒载体. 病毒载体虽然转染效率 高, 但因存在免疫反应等安全性问题, 在临床应用中受 到限制. 非病毒载体因安全性高和易于制备等优势而受 到广泛关注, 但也存在转染效率低、细胞毒性大及细胞 靶向性差等亟待解决的问题. 将嗍酸酯键引入阳离子聚 合物、脂质体等非病毒载体的制备能够改善上述问题, 提高基因输送效率.

冯俊等 ${ }^{[34]}$ 将苯硼酸修饰到低分子量聚乙烯亚胺链 上得到 $\mathrm{PEI}^{1.8 \mathrm{k}}-\mathrm{PBA}_{2.9}$, 与 $\mathrm{PEI}^{1.8 \mathrm{k}}(\mathrm{N} / \mathrm{P}=5)$ 相比, DNA 凝 聚能力得到明显提高, 在 $\mathrm{N} / \mathrm{P}=2.5$ 时即可实现 DNA 全 凝. 随后，将 $\gamma-\mathrm{CD}$ 通过硼酸酯键接枝到 $\mathrm{PEI}^{1.8 \mathrm{k}}-\mathrm{PBA}_{2.9}$ 上 $\left(\mathrm{PEI}^{1.8 \mathrm{k}}-\mathrm{PBA}_{2.9}-\gamma-\mathrm{CD}\right)$, 用于生物模拟细胞表面, 从而 减少血清对转染的负面影响, 并改善载体的生物相容 性. PEI ${ }^{1.8 \mathrm{k}}-\mathrm{PBA}_{2.9}-\gamma-\mathrm{CD}$ 的 $\mathrm{IC}_{50}$ 值比 $\mathrm{PEI}^{25 \mathrm{k}}$ 几乎高 2 倍, 细胞毒性显著降低. 有血清存在时的转染实验表明, $\mathrm{PEI}^{25 \mathrm{k}}$ 和 $\mathrm{PEI}^{1.8 \mathrm{k}}-\mathrm{PBA}_{2.9}$ 的转染效率均随血清的增加而降 低, 而 $\mathrm{PEI}^{1.8 \mathrm{k}}-\mathrm{PBA}_{2.9}-\gamma-\mathrm{CD}$ 在含体积分数为 $10 \%$ 和 $30 \%$

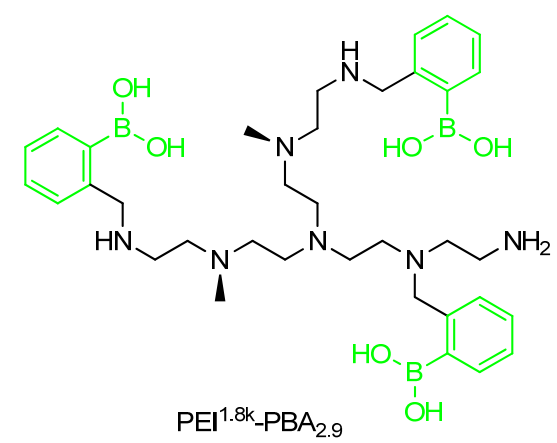

血清的培养基中始终保持高转染效率, 比 $\mathrm{PEI}^{25 \mathrm{k}}$ 高约 25 倍.

采用类似的策略, $\mathrm{Kim}$ 等 ${ }^{[35]}$ 通过还原胺化反应使氧 化的甲基纤维素与聚乙烯亚胺结合(MCPEI), 再引入苯 硼酸后得到阳离子聚合物载体 MCPEI-PBA, 分别通过 噻唑蓝(MTT)实验和苂光素酶基因转染实验测定其在 Hela 细胞、 $2 \mathrm{c} 12$ 细胞和 HepG2 细胞中的细胞毒性和基 因转染效率. MTT 实验表明, 与 PEI 和 MCPEI 相比, MCPEI-PBA 的 $\mathrm{IC}_{50}$ 值明显增大, 证明了其细胞毒性低. 苂光素酶基因转染实验结果显示，在没有血清的情况 下, MCPEI-PBA 在 Hela 细胞和 $\mathrm{c} 2 \mathrm{c} 12$ 细胞中的转染效 率比 MCPEI 和 PEI 低 $5 \sim 8$ 倍, 而在 HepG2 细胞中, MCPEI-PBA 的转染效率比 MCPEI 高 1.4 倍, 比 PEI 高 4.2 倍, 这充分说明了其具有细胞靶向功能. 在有血清 的情况下, MCPEI 和 MCPEI-PBA 的转染效率均低于 PEI, 这是因为氧化的甲基纤维素(MC)链段中的羟基和 甲氧基官能团具有电荷屏蔽作用，从而保证了其血清稳 定性. 而在 HepG2 细胞中, 即使有血清存在的情况下, MCPEI-PBA 的转染效率也比 PEI 高 5.6 倍, 进一步证实 了由 PBA 基团引起的靶向性. 由此可见, 将硼酸酯键引 入基因载体的构筑，能够在保证安全性的前提下有效提 高转染效率.

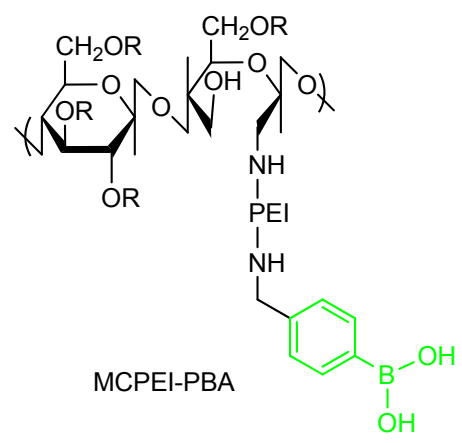

嗍酸衍生物载体还可通过电荷反转实现基因从溶 酶体内逃逸，提高基因转染效率. 申有青等 ${ }^{[36]}$ 制备了一 种依赖于活性氧(ROS)的电荷可逆转聚合物, 聚[(2-丙 烯酰基 $)-$ 乙基-(对喼酸苄基) - 二 乙基溴化铵 $]$ (BPDEAEA). 该聚合物具有强正电荷, 可将 DNA 有效地 包裹到纳米颗粒中，但一旦硣酸基团被细胞内活性氧 (如 $\mathrm{H}_{2} \mathrm{O}_{2}$ ) 氧化, 季铵盐会释放出对羟甲基苯酚并转化为 叔胺. 然后, 叔胺随着酯基自催化水解生成聚丙烯酸, 转变为负电荷. ROS 触发的电荷逆转载体优于快速可生 物降解的载体(例如酸可裂解载体和可生物还原的载 体), 因为所得的带负电荷的聚合物不仅快速释放 DNA, 而且与降解的阳离子片段不同，不干扰基因转录过程. 该新颖的载体基因转染效率高，具有肿瘤靶向能力，抑 制肿瘤细胞生长的能力优于抗癌药物阿需素(DOX), 在 基因治疗领域具有广阔应用前景. 


\section{2 载体类型}

\section{1 药物一聚合物偶联}

药物-聚合物偶联 ${ }^{[37]}$ 是由水溶性聚合物和药物通过 共价键连接在一起的大分子结构, 药物和聚合物上均需 有功能基团. 对于一些含二醇或硼酸成分的药物, 可通 过引入含硼酸或二醇成分的亲水性聚合物制备纳米颗 粒, 既可以使纳米药物有效躲避免疫系统的识别而获得 长时间循环性能, 又能由病变部位的酸性环境引发硼酸 酯键断裂从而将药物释放出来 ${ }^{[38]}$.

穿心莲内酯(AND)及其衍生物具有极高的药用价 值, 如抗氧化作用、抗炎作用、免疫调节活性、抗病毒 作用和抗癌作用等. 但由于其水溶性较差, 临床应用受 到限制. 为了克服这一难题, Kim 等 ${ }^{[39]}$ 利用穿心莲内酯 中存在的二醇能够与嗍酸反应生成喼酸酯键的特性, 构 筑了具有 $\mathrm{pH}$ 和 ATP 双重响应性的药物-聚合物偶联体 系 pPBA-AND, 以提高穿心莲内酯的水溶性(图 6). 他 们将苯硼酸接枝在甲基乙烯基醚一马来酸酐共聚物上形 成聚苯硼酸(pPBA), 然后按物质的量比 $2: 1$ 简单混合 pPBA 和 AND, 制备纳米结构 pPBA-AND, 载药量高达 $34 \%$. 刺激响应性实验表明, $\mathrm{pPBA}-\mathrm{AND}$ 的粒径在 $\mathrm{pH}=$ 5.0 和 $[\mathrm{ATP}]=100 \mu \mathrm{mol} / \mathrm{mL}$ 的条件下发生显著变化, 并
且 AND 的释放量分别达到 $80 \%$ 和 $60 \%$. 然而，在没有 ATP 的中性 $\mathrm{pH}$ 下, AND 的释放量可忽略不计. 此外, 细 胞毒性实验表明 $\mathrm{pPBA}-\mathrm{AND}$ 的 $\mathrm{IC}_{50}$ 值比游离 AND 的 $\mathrm{IC}_{50}$ 值低 5.1 倍. 苂光共聚焦和流式细胞仪结果显示, 细 胞对 pPBA-AND 的摄取量比对游离 AND 的摄取量高 6 倍. 分别给携带 MCF-7 癌细胞的小鼠注射游离 AND 和 pPBA-AND $18 \mathrm{~d}$ 后, 观察到后者的肿瘤体积远小于前 者, 更加明确地阐明了 pPBA-AND 纳米结构具有作为 有效抗癌制剂的潜力.

嗍替佐米(BTZ)是一种用于治疗多发性骨髓瘤的有 效抗癌药物, 但因其副作用极大, 在临床上未能充分实 现价值. 由于㸴替佐米结构内含嗍酸成分, 刘育等 ${ }^{[40]}$ 将 $N$-(2-氨基乙基) - 葡萄糖酰胺 $(\mathrm{Glu})$ 修饰的透明质酸 (HAGlu)与 BTZ 偶联(图 7), 因透明质酸(HA)能够特异 性识别肿瘤细胞表面过量表达的 CD44 受体, 且二者的 连接基团硼酸酯键在肿瘤组织的酸性环境下会断开，由 此赋予嗍替佐米靶向性并减少其毒副作用. Glu-BTZ 在 不同 $\mathrm{pH}$ 值下的 ${ }^{1} \mathrm{H}$ NMR 谱图可以证实其对 $\mathrm{pH}$ 的响应 性. $\mathrm{pH}=5.7$ 时, Glu-BTZ 的谱图与它们各自的谱图相似, 表明二者之间无相互作用，当 $\mathrm{pH}$ 从 6.5 增加到 8.5 时， 可以看到明显的化学位移变化和裂分峰, 并且归属于
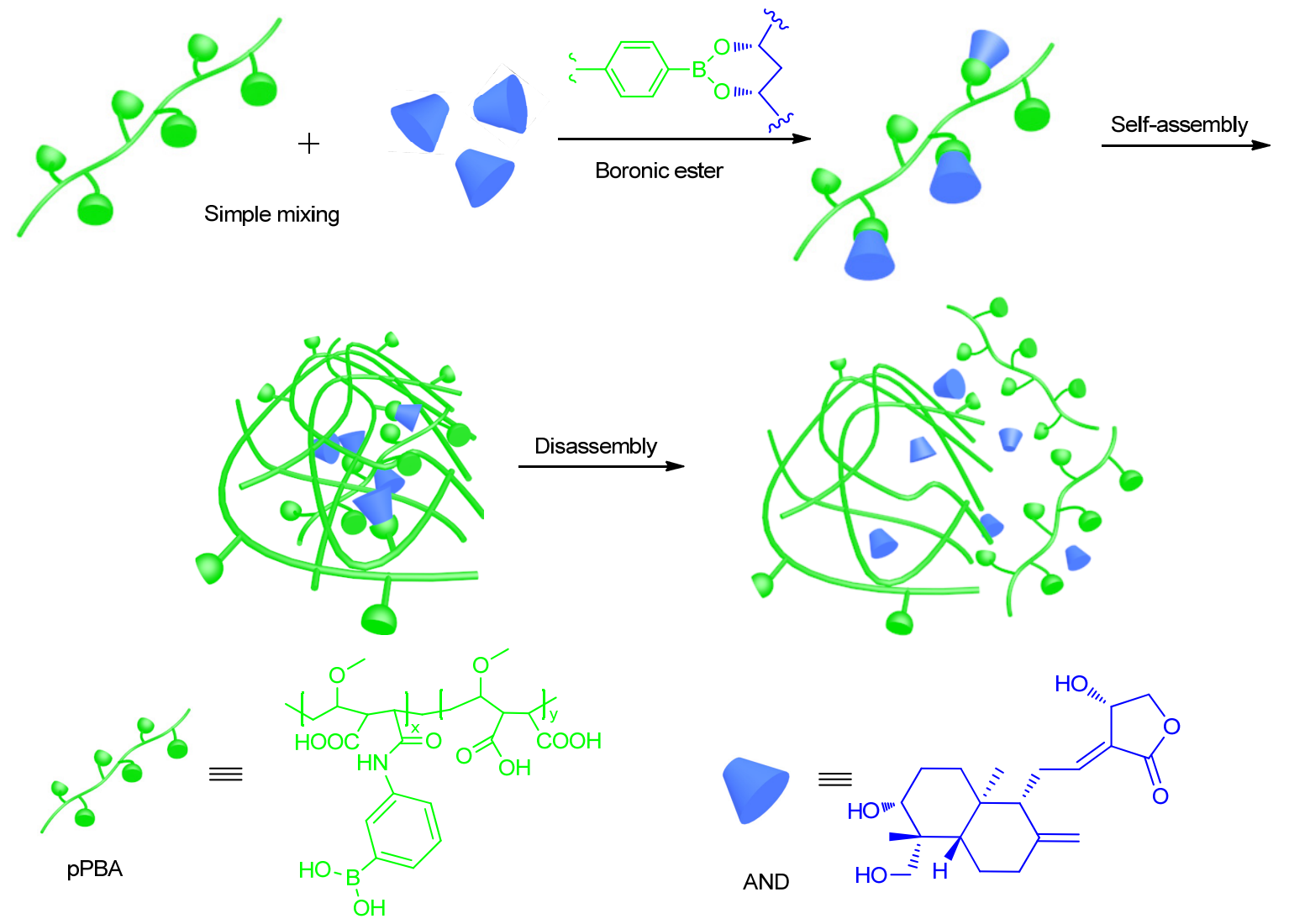

图 6 pPBA-AND 纳米组装体的形成和裂解示意图

Figure 6 Schematic illustration for the formation and destruction of pPBA-AND nanoassembly 


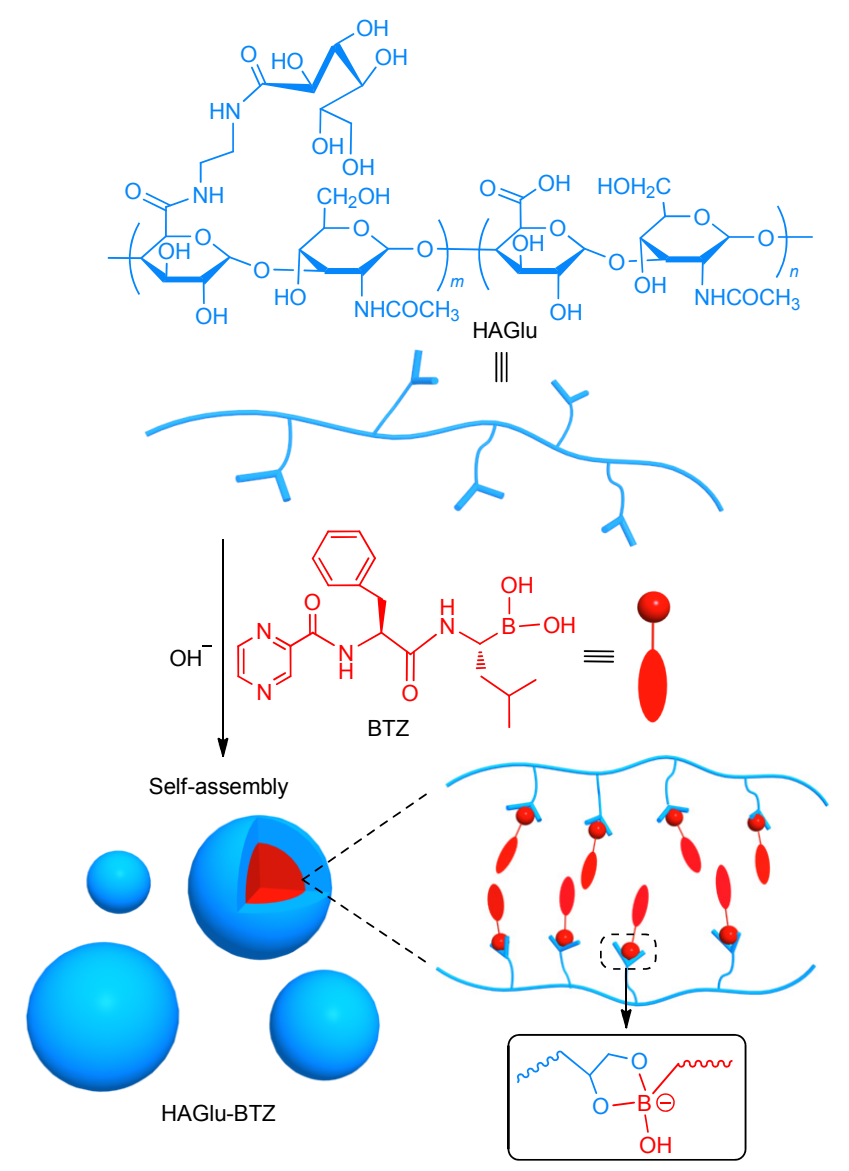

图 7 HAGlu-BTZ 多糖偶联物的构筑示意图 ${ }^{[40]}$

Figure 7 Schematic illustration for the construction of HAGlu-BTZ polysaccharide conjugates
Glu-BTZ 的质子信号 $\mathrm{H}_{\text {Glu-BTZ }}$ 明显增加. 此外, HAG$\mathrm{lu}-\mathrm{BTZ}$ 在不同 $\mathrm{pH}$ 下的药物释放曲线进一步证实了其对 $\mathrm{pH}$ 的响应性. MTT 实验表明, HAGlu-BTZ 可极大改善 BTZ 的疗效, 并降低其毒副作用.

\section{2 聚合物胶束}

聚合物胶束 ${ }^{[41]}$ 是由两亲性共聚物在没有外部干预 的条件下通过其固有的键合位点自组装形成的球形纳 米颗粒. 结构上具有疏水内核和亲水外壳，根据相似相 容原理，其疏水内核可以加载药物，从而增加难溶性药 物的溶解度. 将硼酸酷键引入聚合物胶束的制备, 既可 赋予胶束刺激响应性，又可起到交联作用，降低临界胶 束浓度 $(\mathrm{CMC})$, 提高胶束的稳定性, 延长药物在血液中 的循环时间.

Lam 等 ${ }^{[42]}$ 分别将硝基嗍酸(NBA) 和儿茶酚 (Catechol)引入由聚乙二醇 $\left(\mathrm{PEG}^{5 \mathrm{k}}\right)$ 和树枝状胆酸 $\left(\mathrm{CA}_{8}\right)$ 组 成的嵌段共聚物 $\mathrm{PEG}^{5 \mathrm{k}}{ }_{-} \mathrm{CA}_{8}$ 中, 形成 $\mathrm{PEG}^{5 \mathrm{k}}-\mathrm{NBA}_{4}-\mathrm{CA}_{8}$ 和 $\mathrm{PEG}^{5 \mathrm{k}}$-Catechol ${ }_{4}-\mathrm{CA}_{8}$, 二者通过硼酸酯键的连接形成 交联胶束 $\mathrm{BCM}$ (图 8). 与非交联胶束 $\mathrm{PEG}^{5 \mathrm{k}}-\mathrm{CA}_{8}$ 相比, $\mathrm{BCM}$ 显著降低了临界胶束浓度, 大大提高了胶束在体 内环境中的稳定性, 当暴露于体积分数为 $50 \%$ 的人血浆 $24 \mathrm{~h}$ 后, BCM 仍保持尺寸均匀性, 平均粒径为 $30 \mathrm{~nm}$, 当暴露于十二烷基硫酸钠(SDS) $2 \mathrm{~d}$ 后, 通过动态光散 射仍可观察到恒定的粒径分布, 其自组装结构保持完 整. 此外, BCM 对外界的刺激响应实验表明, 酸性条件 和过量甘露醇可有效破坏硼酸酯键, 在 $\mathrm{pH}=5.0$ 的 SDS

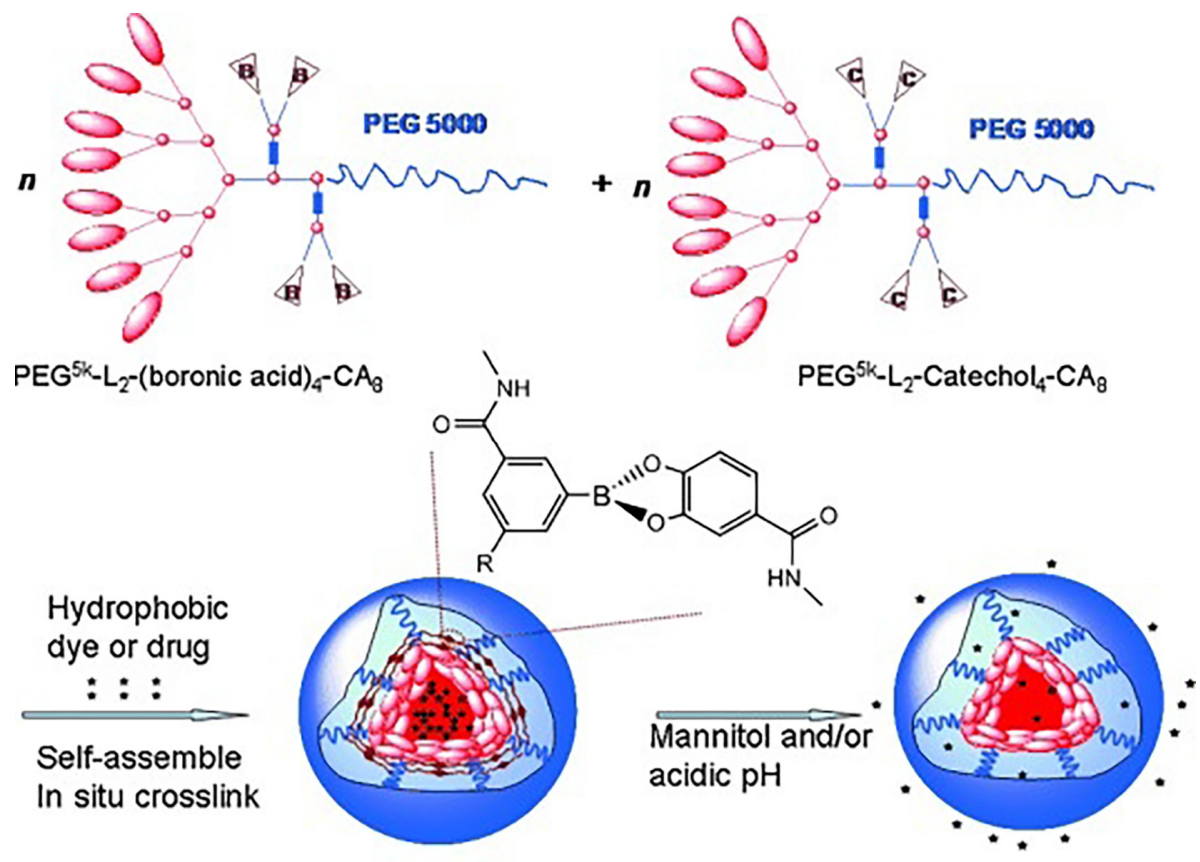

图 8 硼酸盐交联胶束 $(\mathrm{BCM})$ 的形成过程及其响应于甘露醇和/或酸性 $\mathrm{pH}$ 值的疏水染料或药物递送示意图 ${ }^{[42]}$

Figure 8 Schematic illustration of the formation process of the boronate crosslinked micelles (BCM) and its hydrophobic dye or drug delivery in response to mannitol and/or acidic $\mathrm{pH}$ values 
溶液中孵育 $120 \mathrm{~min}$ 后, $\mathrm{BCM}$ 的粒径在 $2 \mathrm{~min}$ 内快速减 小, 在 SDS 和过量甘露醇 $(100 \mathrm{mmol} / \mathrm{L})$ 存在的条件下也 可观察到 $\mathrm{BCM}$ 的粒径快速减少, 而在 SDS 和 100 $\mathrm{mmol} / \mathrm{L}$ 葡萄糖存在的条件下粒径尺寸无明显变化. 透 射电子显微镜(TEM) 显示的胶束结构变化也是如此, 说 明此交联胶束在酸性 $\mathrm{pH}$ 或甘露醇存在下发生响应性裂 解.

葛治伸等 ${ }^{[43]}$ 设计了由聚乙二醇(PEG)和含苯基硼酸 酯的聚甲基丙烯酸酯(PBEMA)组成的 PEG- $b$-PBEMA 胶束, 用于输送疏水性 $\operatorname{Pt}(\mathrm{IV})$ 前药, 通过增加前药的细 胞摄入量和降低细胞内的谷胱甘肽(GSH)浓度来逆转顺 铂耐药性. 中性条件下, 亲水性 PEG 外壳增加了胶束在 血液循环中的稳定性, 当胶束到达酸性肿瘤组织时, 硼 酸酯键断裂, 胶束裂解, 包封的 $\operatorname{Pt}(\mathrm{IV})$ 前药从胶束中有 效释放, 并在癌细胞内 $\mathrm{H}_{2} \mathrm{O}_{2}$ 和抗坏血酸的刺激下还原 成活性顺铂. 小鼠体内抗肿瘤实验表明, 负载前药的胶 束有效逆转了肿瘤组织对顺铂的耐药性.

\section{3 线性-超支化聚合物}

线性一超支化聚合物 ${ }^{[44]}$, 又称星形聚合物或树枝状 聚合物，是指三条或三条以上的线型聚合物链(通常称 为臂)通过有限的结点连接到同一个中心支化点(通常称 为核)而形成的 “星状” 或 “树枝状” 支化聚合物. 传统 聚合物胶束因其两亲性共聚物自组装形成的核壳结构 是药物载体的优先选择. 然而, 因其在体内循环过程中 会被大量的体液和血液高度稀释, 一旦聚合物的浓度低 于临界胶束浓度, 胶束结构就会发生解离; 而永久稳定 的胶束因无法实现药物释放也不受欢迎. 利用硼酸酯键 作为连接结点构筑的超支化聚合物恰好弥补这一缺点.
一方面，超支化聚合物具有大量末端官能团，便于引入 喼酸酯键，所形成的纳米粒子更为稳定. 另一方面，超 支化聚合物分子链缠结程度低，一旦嗍酸酯键在外界刺 激下断裂，纳米粒子的解离更为迅速，有利于药物的释 放. 因此，超支化聚合物在药物递送过程中可以表现出 更好的稳定性和更为灵敏的刺激响应性 ${ }^{[45]}$.

冯俊等 ${ }^{[46]}$ 利用高度支化的聚碳酸酯(HEHDO) 与苯 嗍酸修饰的聚乙二醇(mPEG-PBA)之间形成的硼酸酯 键，构筑了具有多臂星形结构的线性超支化纳米结构 mPEG-PBA/HEHDO(图 9). 在不同 $\mathrm{pH}$ 下混合 $\mathrm{mPEG}-$ PBA 和 HEHDO, 通过 UV 光谱监测 mPEG-PBA/ HEHDO 在酸性条件下的结构变化, 结果显示在 $\mathrm{pH}=$ 7.4 时, 最大紫外吸收波长在 $272 \mathrm{~nm}$, 表明硼酸酯键的 快速形成, 而 $\mathrm{pH}=4.0$ 时最大紫外吸收波长在 $266 \mathrm{~nm}$, 与 mPEG-PBA 相同, 表明没有形成嗍酸酯键. 表明在 $\mathrm{pH} 4.0 \sim 7.4$ 范围内, $\mathrm{pH}$ 值越高, 纳米结构越易形成. 加 载模型药物 DOX 后, mPEG-PBA/HEHDO 作为药物载体 显示出潜在的应用价值，对 DOX 的负载率为 $16 \%$, 且 当 $\mathrm{pH}=7.4$ 时，在 $120 \mathrm{~h}$ 内仅有不到 $10 \%$ 的 DOX 释放， 而当 $\mathrm{pH}=5.0$ 时, $\mathrm{DOX}$ 在 $70 \mathrm{~h}$ 内的释放量接近 $100 \%$ ，且 在存在体积分数为 $10 \%$ 的血清或在 $1 \mathrm{mg} / \mathrm{mL}$ 的典型血 糖水平的条件下尺寸无明显变化.

危岩等 ${ }^{[47]}$ 利用 Boltorn H40 (H40)与喼酸封端的甲 氧基聚乙二醇 $\left(\mathrm{mPEG}-\mathrm{B}(\mathrm{OH})_{2}\right)$ 、喼酸功能化的 AIE 染料 $\left(\mathrm{PhB}(\mathrm{OH})_{2}\right)$ 之间形成的硣酸酯键制备了一种具有聚集 诱导发光性质的纳米粒子 H40-star-mPEG-PhB $(\mathrm{OH})_{2}$. 该纳米粒子是一种两亲性树枝状大分子聚合物，高度支 化的结构大大提高了其对 AIE 染料的负载率，使得细胞
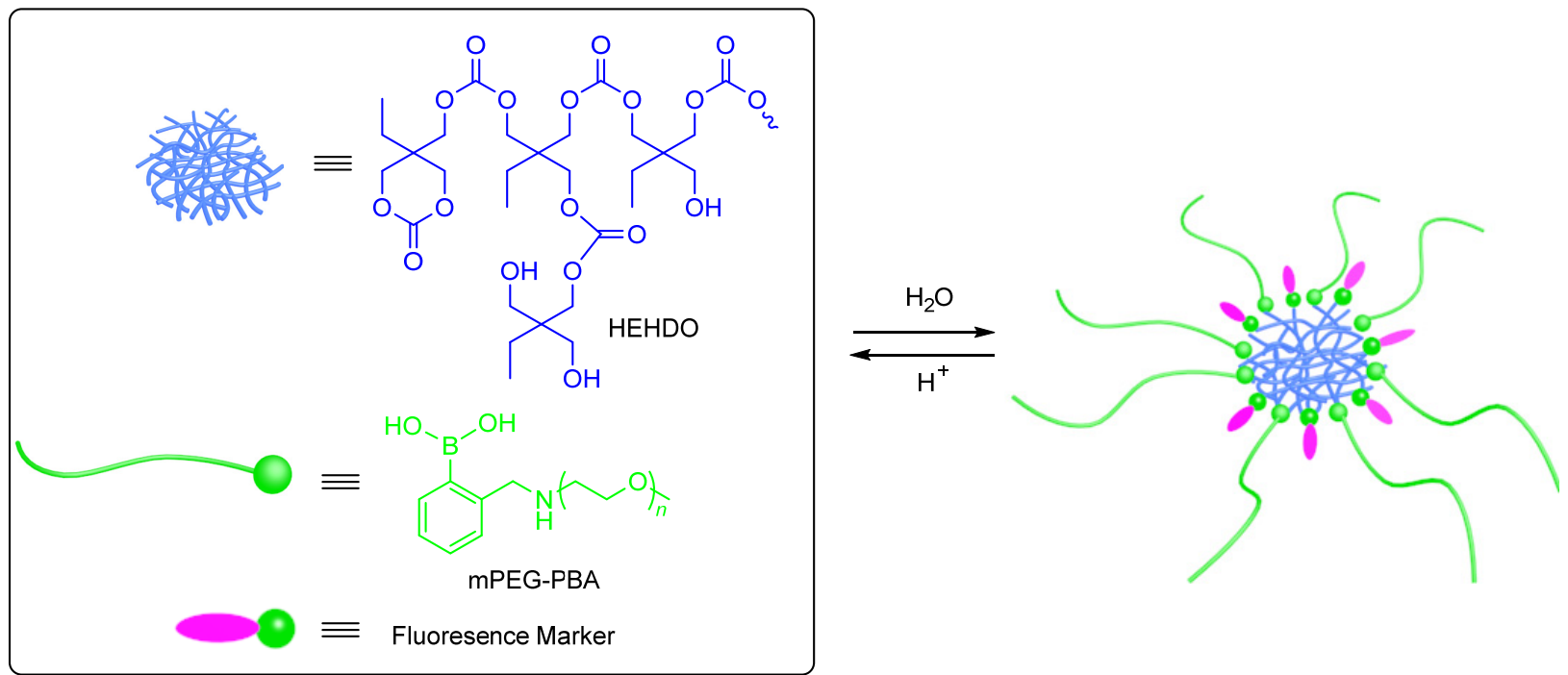

图 9 动态稳定的 mPEG-PBA/HEHDO 纳米结构的形成过程示意图

Figure 9 Schematic illustration of the formation process of dynamically stable mPEG-PBA/HEHDO nanoconstruction 
成像更加清晰明亮. 因为超支化及高负载能力, 该纳米 粒子也可作为大量疏水性药物的储库, 为药物递送提供 了新的载体.

此外, 当超支化聚合物用作基因载体时, 引入苯嗍 酸既可利用硼酸与核酸之间形成的硼酸酯键增加载体 与基因的结合力，也可通过硼酸与氨基之间的硼氮配位 键以及苯环的疏水性促进超支化聚合物压缩为更紧凑 的小聚集体，能够在保持阳离子聚合物较低毒性的同时 实现高效的核酸递送, 从而解决基因递送过程中转染效 率与细胞毒性之间的矛盾, 实现 “高效低毒” 的基因递 送. 如程义云等 ${ }^{[48]}$ 利用苯嗍酸修饰的分子量仅为 3256 的第二代聚酰胺树形高分子(G2 PAMAM), 实现了 DNA 和 siRNA 的高效递送.

\section{4 介孔二氧化硅}

介孔二氧化硅纳米粒子(MSN) 是一种孔径介于 2 $50 \mathrm{~nm}$ 的无机多孔材料, 与有机高分子材料相比, 具有 物理化学性质稳定、无毒且生物相容性好等优点, 是一 种良好的药物输送载体 ${ }^{[49]} .2001$ 年, Regi 等 ${ }^{[50]}$ 首次将 MSN 用作药物载体, 以布洛芬为模型药物证明了 MSN 可通过介孔通道及其内部空腔实现药物的负载与释放. 此外，介孔二氧化硅纳米粒子表面富含硅羟基，易于对 其表面进行功能化修饰. 研究表明, 如果不对 MSN 表 面进行修饰，药物在血液循环过程中容易渗漏，因此， 构筑基于 MSN 的刺激响应性药物输送体系成为研究的 热点 ${ }^{[51]}$. 将硼酸酯键引入 MSN 载体, 作为药物释放的
开关, 可构筑响应于酸性 $\mathrm{pH}$ 和高葡萄糖浓度的药物载 体.

王新灵等 ${ }^{[52]}$ 将苯硼酸接枝到 MSN 表面, 利用硼酸 与二醇之间发生的酯化反应将单宁覆盖在 MSN 表面 (Tannin-MSN)作为封孔剂以防止药物外渗. 实验证明, Tannin-MSN 在 $\mathrm{pH}=5$ 的酸性条件下可在 $20 \mathrm{~h}$ 内释放出 $80 \%$ 的药物，而在中性条件下的药物释放量几乎为 0 , 极大地提高了药物利用率. 这是因为, 在中性条件下, 硼酸酯键稳定存在，单宁将 MSN 表面的孔道封住，防 止药物非特异性渗漏, 而在酸性条件下, 硼酸酯键断裂, 单宁与 MSN 之间的连接断裂, 开关打开, 实现药物可 控释放.

采用类似的原理，刘传军等 ${ }^{[53]}$ 通过酰胺缩合反应 将葡萄糖胺接枝到聚丙烯酸(PAA)修饰的 MSN 表面，并 用乙二胺桥联苯硼酸对其进行交联，以罗丹明 $6 \mathrm{G}$ $(\mathrm{Rd} 6 \mathrm{G})$ 为模型药物研究了其 $\mathrm{pH}$ 响应的药物释放行为 (图 10). 将装载 $\mathrm{Rd} 6 \mathrm{G}$ 的 $\mathrm{MSN}$ 在 $\mathrm{pH}=7.4$ 的 $\mathrm{PBS}$ 溶液 中孵育 $40 \mathrm{~h}$ 后，测得 $\mathrm{Rd} 6 \mathrm{G}$ 的释放量仅为 $2 \%$ ，可视为零 过早释放, 反映了聚合物封端的 MSN 系统对药物的优 异阻断性能. 而在 $\mathrm{pH}=6.0$ 的条件下捊育 $40 \mathrm{~h}$ 后, $\mathrm{Rd} 6 \mathrm{G}$ 的释放量为 $45 \%, 189 \mathrm{~h}$ 后累计释放量高达 $84 \%(\mathrm{pH}=$ 7.4 的对照组为 $3.4 \%$ ), 药物利用率较高. 该研究表明, $\mathrm{MSN}$-聚合物体系是一种具有潜在前景的刺激响应性药 物载体

杨英威等 ${ }^{[54]}$ 报道了一种具有超顺磁性 $\mathrm{Fe}_{3} \mathrm{O}_{4}$ 核和 MSN 壳的纳米载体, MSN 壳表面用 4-羧基苯硼酸加以
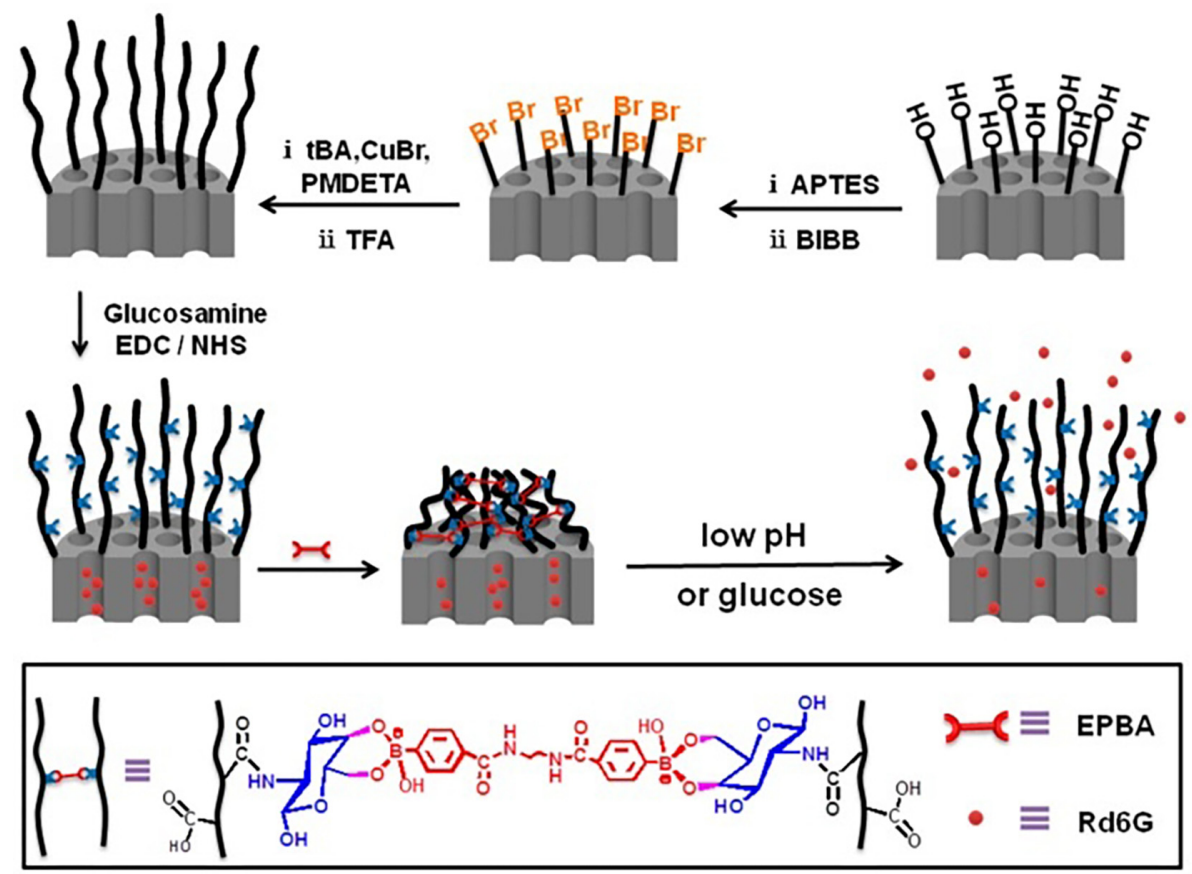

图 10 交联 MSN-PAA-AGA 的制备及其双重响应药物释放过程的示意图 ${ }^{[53]}$

Figure 10 Schematic illustration of the preparation of cross-linked MSN-PAA-AGA and its dual-responsive drug release process 
修饰, 并通过路酸与 $\beta$-环糊精反应生成的硼酸酯键在外 表面共价安装 $\beta$-环糊精层, 作为二氧化硅纳米容器的 “门”。该纳米复合材料具有均匀的尺寸、优异的分散性、 有序的介孔性和强磁化强度. 实验证明, $\mathrm{Fe}_{3} \mathrm{O}_{4} @ \mathrm{MSN} @$ $\beta-\mathrm{CD}$ 具有良好的 $\mathrm{pH}$ 和糖响应特性, 可通过调节 $\mathrm{pH}$ 值 和提高 $D$-果糖浓度来实现药物的持续释放, 而且细胞 毒性低, 能够有效地将药物转运到目标部位.

\section{3 药物与载体结合的方式}

\section{1 共价键结合}

含有喼酸或二醇成分的药物可直接通过硼酸酯键 与载体连接, 嗍酸酯键断裂即可实现药物的释放, 通过 共价键作用加载药物的载体通常具有较高的载药率, 如 药物-聚合物偶联, 这种结合方式较为牢固, 一般在体 循环过程中不会发生药物的渗漏.

阿霉素(Dox)是一种适用于多种癌症的广谱抗癌药, 虽然已有多种载体用于减少其副作用, 但因载药量有限 未能达到理想效果. 最近, 史文等 ${ }^{[55]}$ 制备了一种硅烷一 碍酸前体(图 11), 它不仅含有用于形成有机硅纳米颗粒 的烷氧基硅烷, 而且还含有与二醇化合物反应的苯嶰 酸. 利用该化合物, 可以有效地实现在制备有机硅纳米 颗粒过程中对 Dox 共价负载, 载药量可达 $22.4 \%$. 透明 质酸(HA)的进一步修饰使 Si-Dox@HA 纳米颗粒具有靶 向 CD44 过表达癌细胞的能力. Si-Dox@HA 纳米粒子表 现出 $\mathrm{H}_{2} \mathrm{O}_{2}$ 响应的控释能力, 药物释放量高达到 $80 \%$, 能 够实现药物的充分利用.

卡培他滨(CAPE), 是一种含 1,2-二醇的抗癌药物.
Herrera-Alonso 等 ${ }^{[56]}$ 通过喼酸酯键的形成将卡培他滨与 由聚乙二醇和苯嗍酸功能化的聚碳酸酯组成的嵌段共 聚物 $\mathrm{PEG}_{44}-\mathrm{b}-\mathrm{PPBC}_{26}$ 连接在一起，接枝率可达 $75 \%$ ，与 被频哪醇保护的不能与 CAPE 发生酯化反应的 PEG $_{44-}$ $\mathrm{b}-\mathrm{PPBC}_{26}$ 相比, 硼酸酯键的形成大大提高了载药量和载 药效率. CAPE-PEG $44-b-P-P B C_{26}$ 的药物释放曲线表明, 当聚合物分别在 $\mathrm{pH}$ 为 7.4, 6.5, 5.5 的 PBS 溶液中孵育 $24 \mathrm{~h}$ 后, 其药物释放量从 $17 \%$ 增加到 $85 \%$. 这一结果证 实了此药物-聚合物共轭体系对于 $\mathrm{pH}$ 的敏感性及其作 为药物递送载体的巨大潜能.

\section{2 物理包封作用}

对于不含功能基团(二醇和硼酸)的药物, 可通过物 理包封作用加载到含有硼酸酯键的载体中, 药物释放由 硼酸酯键断裂引起的载体解离间接实现. 通过物理包封 作用加载药物的载体通常制备过程简单，可通过 “一锅 法” 同时实现胶束的制备和药物的负载.

张灿等 ${ }^{[57]}$ 提出了基于硼酸-二醇的一步式模块化组 装策略, 将二醇改性的亲水性结构单元多巴胺共轭聚合 物(Poly-DA)、嗍酸改性的疏水性结构单元苯硼酸修饰脱 氧胆酸(PBA-DOCA)和模型药物疏水性阿霉素(DOX)简 单地溶解在一起，在中性生理条件下自组装形成具有 核/壳结构包封药物的胶束, 其载药量为 $22.4 \%$. 胶束在 $\mathrm{pH}=7.4$ 时的 ${ }^{1} \mathrm{H}$ NMR 谱图显示, PBA 苯环上的化学位 移 $\delta$ 从 7.80 7.87 变为 7.50 7.73, 而 DA 的化学位移 $\delta$ 从 $6.70 \sim 6.85$ 变为 $6.53 \sim 6.58$, 表明硼酸酯键的存在. 将其在含有 $10 \mathrm{mmol} / \mathrm{L}$ 葡萄糖和体积分数为 $10 \%$ 的胎牛 血清(FBS)的 $\mathrm{pH}=7.4$ 的缓冲溶液中孵育 $24 \mathrm{~h}$ 后, DOX

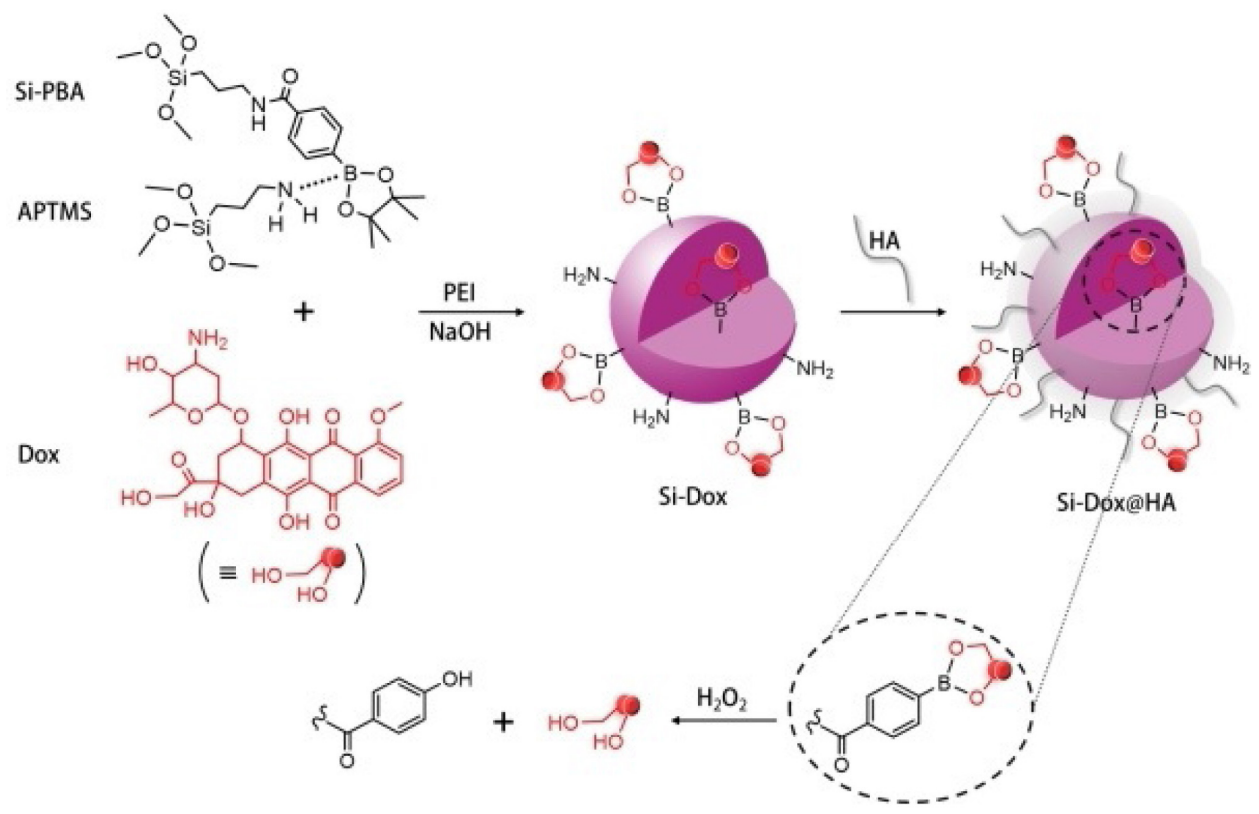

图 11 Si-Dox@HA 纳米结构的制备及其用于 $\mathrm{H}_{2} \mathrm{O}_{2}$ 响应的 Dox 释放过程示意图 ${ }^{[55]}$

Figure 11 Schematic illustration of preparation of Si-Dox@HA nanoconstruction and its $\mathrm{H}_{2} \mathrm{O}_{2}$-responsive Dox release process 
的释放量仅为 $20 \%$, 动态光散射(DLS)也未观察到尺寸 分布的显著变化. 而在 $\mathrm{pH}=5.5$ 的环境中, ${ }^{1} \mathrm{H}$ NMR 谱图 与两种单独化合物的叠加谱图相似, 表明 PBA 和 DA之 间未形成硼酸酯键; DLS 显示粒径从 $90 \mathrm{~nm}$ 增加到 194.2 $\mathrm{nm}$, DOX 在 $24 \mathrm{~h}$ 内的释放量由 $20 \%$ 增加到 $60 \%$. 这些 结果充分表明 DOX 的释放是由硼酸酯键在酸性条件下 的断裂导致胶束解离而引发的.

汤琪云等 ${ }^{[58]}$ 分别将 3-氨基苯硼酸和 3,4-二羟基苯乙 胺接枝到修饰有四苯乙烯的聚乙二醇- $b$-聚 $L$-谷氨酸苄 酯的嵌段共聚物上(图 12), 通过二者之间嗍酸酯键的制 备了一种多功能核交联胶束(CCLMs), 用于抗癌药物依 托泊苷(ETO)的递送和生物成像. 依托泊苷通过物理包 封作用加载到胶束的疏水性核中, 负载率和包封率分别 为 $8.2 \%$ 和 $83.1 \%$. 由于二硫键和硼酸酯键的存在, 该胶 束具有低 $\mathrm{pH}$ 值和高谷胱甘肽浓度双重刺激响应性, 在 $\mathrm{pH}=5.0$, 谷胱甘肽浓度为 $50 \mu \mathrm{mol} / \mathrm{mL}$ 的环境下可实现 $84.1 \%$ 的药物释放率. 同时, 由于酸性和还原性是肿瘤 溶酶体的典型条件, 该胶束在递送抗癌药物时具有被动 靶向性. 此外, 由于四苯乙烯(TPE)是一种典型的聚集 诱导发光(AIE)生色团 ${ }^{[59]}$, 该胶束具有生物成像作用. 实验表明, 嗍酸酯键的形成限制了 TPE 分子的分子内旋 转, 增强了胶束的抗光猝灭性. 因此, 该胶束在肿瘤治 疗方面具有巨大潜力.

\section{3 静电作用}

核酸分子内有大量的带负电荷的磷酸根, 而阳离子 聚合物表面有大量的正电荷, 二者通过正负电荷间的静 电作用结合在一起. 嗍酸酯键断裂时, 阳离子聚合物解 体导致其表面的正电荷减少, DNA 与载体的静电作用由 此减弱, 从而实现 DNA 的释放.

高钟镐等 ${ }^{[60]}$ 分别用儿茶酚(Cat) 和苯硼酸(PBA)修饰 聚乙二醇(PEG)和聚乙烯亚胺(PEI), 通过 PBA 和 Cat 之 间形成硼酸酯键构筑了一种用于递送 siRNA 的纳米粒 子 PEG-CPB-PEI. 因为 PEI 表面带有正电荷, 可以与 siRNA 的阴离子磷酸基团产生静电作用, 因此在 $\mathrm{N} / \mathrm{P}=$ 15 时 PEG-CPB-PEI 就能够完全凝聚 siRNA, 且 PEGCPB-PEI/siRNA 的 zeta 电位为 $6 \mathrm{mV}$, 说明纳米颗粒表面 几乎呈电中性, 证明了 PEG-CPB-PEI 对 siRNA 的负载 能力. 在中性环境下, 硼酸酷键稳定存在, PEG 壳的引 入可在体循环过程中 “屏蔽” PBA 配体以减少其 “脱靶 效应” 并降低 PEI 的毒性. 在酸性环境下, 嶰酸酯键断 裂, PEG 壳脱落, 暴露的 PBA 部分与癌细胞上过表达的 唾液酸结合, 从而通过唾液酸受体介导的摄入和延长在 细胞表面的停留时间，增强了细胞的内化吸收.

Kim 等 ${ }^{[61]}$ 分别将苯硼酸(PBA)和半乳糖(Gal)引入聚 乙烯亚胺(PEI)中, 通过 PBA 与 Gal之间硼酸酯键的形成 对 PEI 进行交联, 得到高分子量 PEI, 并以血管内皮生

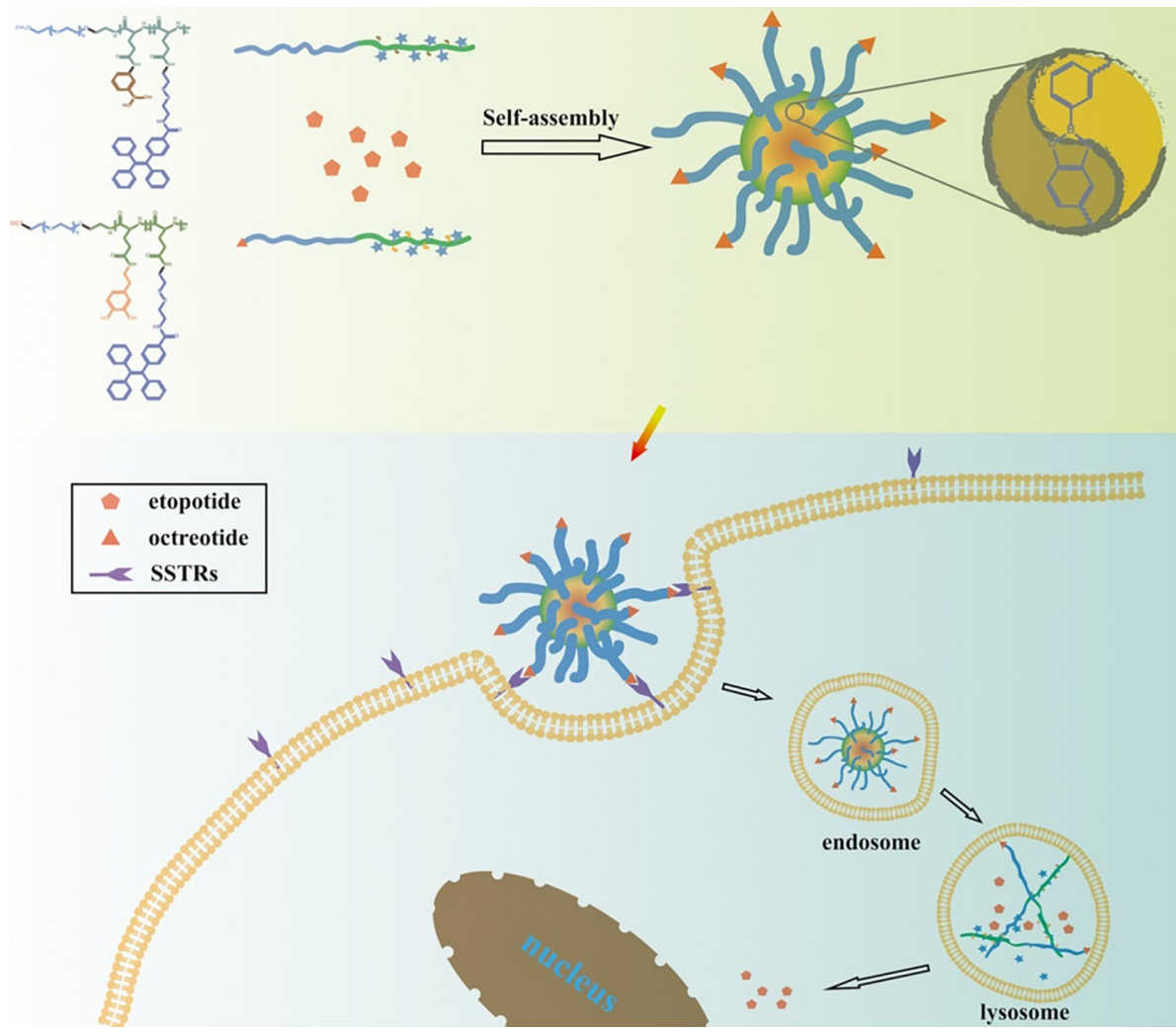

图 12 装载 ETO 的 CCLMs 的形成过程及其用于 $\mathrm{pH}$ 和氧化还原响应的 ETO 递送示意图 ${ }^{[58]}$

Figure 12 Schematic illustration of formation process of ETO-loaded CCLMs and its $\mathrm{pH}$ - and Redox-responsive ETO delivery 
长因子受体(sFlt-1)作为报告基因, 研究了其基因输送能 力. 琼脂糖凝胶电泳、体外转染试验及苂光共聚焦均表 明交联后的 PEI 比未交联的 PEI, PBA-PEI 和 Gal-PEI 有 更高的转染效率, 且在酸性 $\mathrm{pH}$ 下能够实现 DNA 的有效 释放. 这与交联后的 PEI 具有更高的电荷密度及 PBA 部 分赋予的靶向性密切相关.

\section{4 硼酸酯键断裂机制}

目前, 用于药物载体中的硼酸酯键有两种断裂机 制. 第一种是由酸性 $\mathrm{pH}$ 引发的 ${ }^{[22-63]}$. 众所周知, 硼酸酯 键是由硼酸和二醇通过酯化反应形成, 而酯化反应是可 逆的，在酸性条件下，反应会向最初的反应物方向移动， 硼酸酯键由此断裂. 硼酸酯键的另一种断裂机制是由葡 萄糖、甘露醇、过氧化氢 $\left(\mathrm{H}_{2} \mathrm{O}_{2}\right)$ 和三磷酸腺苷(ATP)等含 二醇基团的物质与嗍酸酯键中的二醇发生竞争性反应 导致的 ${ }^{[64-65]}$. 在设计药物载体过程中, 既可利用其中一 种机制, 由单一刺激引发药物释放, 也可同时使用两种 机制，制备多刺激响应性药物载体.

近年来, 过氧化氢诱导嗍酸酯键水解的特性在载药 领域得到了广泛的应用, 并展示出了一些独特的性质. 据报道, 癌细胞的活性氧(ROS)浓度是正常细胞的 10 倍 ${ }^{[66]}$, 朱新远等 ${ }^{[67]}$ 利用这一特点制备了一种循环肿瘤 细胞(CTC)检测纳米探针, 该探针含有苯硼酸频哪醇酯, 可以快速响应 CTC 的高水平内源性 $\mathrm{H}_{2} \mathrm{O}_{2}$ 并通过荧光发 射报告信号, 克服了传统的 CTC 计数分析存在的假阴 性结果问题. 过量的 ROS 在多种疾病(包括炎性疾病、 传染病、心血管疾病、神经退行性疾病、糖尿病和癌症) 的发生和发展中起着重要作用, 张建祥等 ${ }^{[68]}$ 已证明含 芳基硼酸酯的纳米颗粒(NPs)可通过有效地消耗患病组
织中异常增加的 $\mathrm{H}_{2} \mathrm{O}_{2}$ 来消灭炎症. 另一方面, 当 ROS 消除系统不能有效消耗过量的 $\mathrm{H}_{2} \mathrm{O}_{2}$ 时, 超过某个阈值 的过高的 ROS 水平将通过氧化应激作用选择性杀死癌 细胞 ${ }^{[69]}$. 根据这一特点, 唐建斌团队 ${ }^{[70]}$ 和葛治伸团队 ${ }^{[71]}$ 分别将能够促进癌细胞产生 ROS 的 $\beta$-拉帕酮(Lapa)和 棕榈酸抗坏血酸酯 $(\mathrm{PA})$ 引入含嗍酸酯键的药物载体中 以提高肿瘤部位的 $\mathrm{H}_{2} \mathrm{O}_{2}$ 浓度, 制备能够产生协同作用 的纳米颗粒. 纳米粒子被细胞内吞后, 首先释放 Lapa 或 $\mathrm{PA}$, 使肿瘤部位的 $\mathrm{H}_{2} \mathrm{O}_{2}$ 浓度显著提高. 过量的 $\mathrm{H}_{2} \mathrm{O}_{2}$ 一 方面触发嗍酸酯键的断裂以促进抗癌药物或能够抑制 肿瘤细胞抗氧化能力的醌甲基化物的释放, 增加药物载 体的响应灵敏性; 另一方面, 过量的 $\mathrm{H}_{2} \mathrm{O}_{2}$ 本身对癌细 胞具有选择性杀伤作用, 可与抗癌药或醌甲基化物起到 协同作用. 此外, 由于 Lapa 和 PA 在正常细胞中引起的 ROS 升高不明显，从而阻滞了抗癌药物或醌甲基化物 在血液循环过程中的释放, 能够降低细胞毒性并提高药 物利用率. 实验表明, 这种新颖的能够产生协同作用的 纳米颗粒可显著增强治疗功效, 并有可能转化为临床应 用. 随后, Stang 等 ${ }^{[72]}$ 结合自组装和组装后修饰方法, 开 发了一种能够封装 PA 和 DOX 的自组装体 Pt NPs, 它由 有机铂金属环中心和三个 $\mathrm{H}_{2} \mathrm{O}_{2}$ 反应性二嵌段共聚物臂 组成. 实验表明, 通过将化学疗法和氧化应激作用以协 同方式结合起来, Pt NPs@PA/DOX 具有出色的抗肿瘤 功效. 该研究为合理设计基于超分子集合体 ${ }^{[73]}$ 的新药 物递送系统提供了有价值的方法和信息.

江国华等 ${ }^{[74]}$ 将苯硼酸频哪醇酯(PBEM)引入到含有 苯嗍酸的两亲性嵌段共聚物上(图 13)，基于 PBEM 和 $\alpha-\mathrm{CD}$ 之间的主客体作用制备了一种具有葡萄糖和 $\mathrm{H}_{2} \mathrm{O}_{2}$ 双刺激响应性的聚合物囊泡. 以阿需素(DOX)为模型药

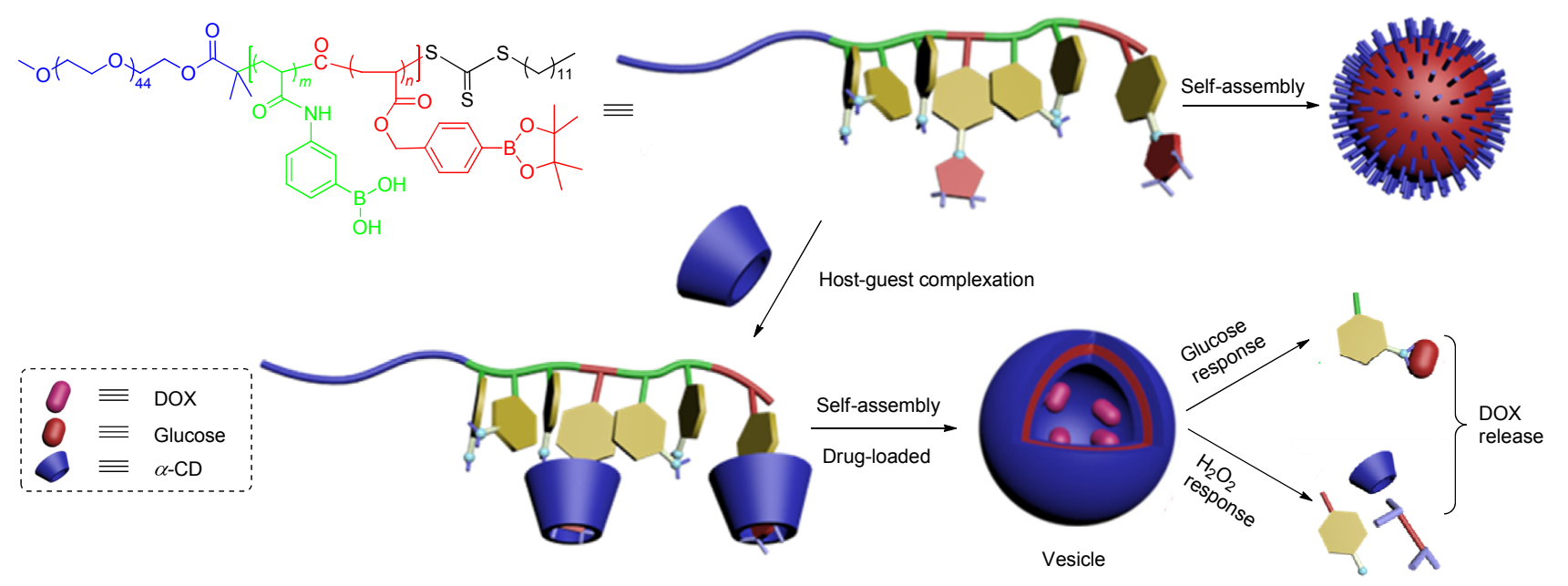

图 13 基于 $\alpha-\mathrm{CD}$ 和 PBEM 的主客体复合物的形成及其用于双重刺激响应的药物释放过程示意图

Figure 13 Schematic illustration of formation of host-guest complexation between $\alpha$-CD and PBEM as well as its dual stimuli-responsive drug release process 
物, 证实了其对葡萄糖和过氧化氢的刺激响应释放行 为. 在没有外部刺激的情况下, DOX 在 $24 \mathrm{~h}$ 内的释放量 低于 $20 \%$, 当葡萄糖浓度为 $11 \mathrm{mmol} / \mathrm{L}$ 时, DOX 在 $6 \mathrm{~h}$ 内的释放水平达到 $70 \%$, 在浓度为 $0.5 \mathrm{mmol} / \mathrm{L}$ 的 $\mathrm{H}_{2} \mathrm{O}_{2}$ 的刺激下, DOX 的释放在 $1 \mathrm{~h}$ 内即可完成, 最终释放率 达到 $80 \%$. 因肿瘤细胞内的葡萄糖浓度和 $\mathrm{H}_{2} \mathrm{O}_{2}$ 浓度均 高于正常细胞, 所以该聚合物囊泡可利用葡萄糖和 $\mathrm{H}_{2} \mathrm{O}_{2}$ 双刺激实现抗癌药物的高效递送.

\section{5 结论}

综上所述, 硼酸酯键作为一种动态共价键, 在构筑 刺激响应性药物载体中具有重要作用, 它解决了由共价 键构筑导致的药物不易释放和非共价键构筑引起的体 循环稳定性差的难题. 硼酸酯键既可用于抗癌药物和胰 岛素的输送，也可用于基因的递送; 既可作为药物与载 体的连接基团，也可作为交联剂提高载体的稳定性; 既 可用于聚合物-药物偶联和聚合物胶束的制备, 也可引 入多孔二氧化硅中. 尽管如此, 嗍酸酯键在刺激响应性 药物载体构筑中仍然面临许多挑战, 如硼酸酯键骤然断 裂必然引起药物突释, 从而导致局部药物浓度过高及药 效维持时间过短. 此外, 作为一种对生物体内葡萄糖、 三磷酸腺苷(ATP) 和过氧化氢 $\left(\mathrm{H}_{2} \mathrm{O}_{2}\right)$ 等多种内源性分子 高度敏感的刺激响应位点, 如何避免其它生物分子的潜 在干扰和竞争效应以实现对特定成分的响应是硼酸酯 键在刺激响应性载体构筑方面面临的最大挑战. 药物缓 释体系可以减少给药次数, 保证血药浓度平稳, 实现药 物的定位、定时及定速释放, 今后的研究重点可转向将 硼酸酯键引入药物缓释体系的设计, 并引入其它功能模 块，构筑集生物检测、生物成像及药物递送于一体的多 功能载体.

\section{References}

[1] Sun, Q. H.; Sun, X. R.; Ma, X. P.; Zhou, Z. X.; Jin, E. L.; Zhang, B.; Shen, Y. Q.; Van Kirk, E. A.; Murdoch, W. J.; Lott, J. R.; Lodge, T. P.; Radosz, M.; Zhao, Y. L. Adv. Mater. 2014, 26, 7615.

[2] Peer, D.; Karp, J. M.; Hong, S.; FaroKHzad, O. C.; Margalit, R.; Langer, R. Nat. Nanotechnol. 2007, 2, 751 .

[3] Ma, X. H.; Wang, J. Y.; Guo, J. J.; Wang, Z. Y.; Zang, S. Q. Chin. J. Chem. 2019, 37, 1120.

[4] Meng, F. D.; Sun, J.; Li, Z. B. Chin. J. Chem. 2019, 37, 1137.

[5] Guan, X. L.; Wang, L.; Li, Z. F.; Liu, M. N.; Wang, K. L.; Lin, B.; Yang, X. Q.; Lai, S. J.; Lei, Z. Q. Acta Chim. Sinica 2019, 77, 1036 (in Chinese).

(关晓琳, 王林, 李志飞, 刘美娜, 王凯龙, 林斌, 杨学琴, 来守 军, 雷自强, 化学学报, 2019, 77, 1036.)

[6] Rowan, S. J.; Cantrill, S. J.; Cousins, G. R. L.; Sanders, J. K. M.; Stoddart, J. F. Angew. Chem., Int. Ed. 2002, 41, 898.

[7] Zhang, Y. L.; Yang, B.; Xu, L. X.; Zhang, X. Y.; Tao, L.; Wei, Y. Acta Chim. Sinica 2013, 71, 48 (in Chinese). (张亚玲, 杨斌, 许亮金金, 张小勇, 陶否, 危岩, 化学学报, 2013, $71,48$.

[8] Tang, Q. Q.; Yuan, L.; Yang, D.; Hu, J. H. Acta Chim. Sinica 2010,
68, 1925 (in Chinese)

(唐倩倩, 袁丽, 杨东, 胡建华, 化学学报, 2010, 68, 1925.)

[9] Xu, H. P.; Cao, W.; Zhang, X. Acc. Chem. Res. 2013, 46, 1647.

[10] Brooks, W. L. A.; Sumerlin, B. S. Chem. Rev. 2016, 116, 1375.

[11] Vancoillie, G.; Hoogenboom, R. Polym. Chem. 2016, 7, 5484.

[12] Lorand, J. P.; Edwards, J. O. J. Org. Chem. 1959, 24, 769.

[13] Bosch, L. I.; Fyles, T. M.; James, T. D. Tetrahedron 2004, 60, 11175 .

[14] Ma, R. J.; Shi, L. Q. Polym. Chem. 2014, 5, 1503.

[15] Djanashvili, K.; Frullano, L.; Peters, J. A. Chem.-Eur. J. 2005, 11, 4010.

[16] Dick, L. R.; Fleming, P. E. Drug Discovery Today 2010, 15, 243.

[17] Kupperman, E.; Lee, E. C.; Cao, Y. Y.; Bannerman, B.; Fitzgerald, M.; Berger, A.; Yu, J.; Yang, Y.; Hales, P.; Bruzzese, F.; Liu, J.; Blank, J.; Garcia, K.; Tsu, C.; Dick, L.; Fleming, P.; Yu, L.; Manfredi, M.; Rolfe, M.; Bolen, J. Cancer Res. 2010, 70, 1970.

[18] Wang, C.; Hou, X. B.; Fang, H. Acta Pharm. Sin. 2019, 54, 1940 (in Chinese).

(王川，侯旭奔，方浩，药学学报, 2019, 54, 1940.)

[19] Zhu, S.-S.; Chen, X.; Yan, Q. Y.; Lin, Z. Chin. Mod. Doctor 2019 , 57,164 (in Chinese) (朱珊珊, 陈傘金, 颜巧妍，林忠，中国现代医生， 2019, 57, 164.)

[20] Escalante, J.; McQuade, R. M.; Stojanovska, V.; Nurgali, K. Maturitas 2017, 105, 23.

[21] Zhu, J. Y.; Lei, Q.; Yang, B.; Jia, H. Z.; Qiu, W. X.; Wang, X. L.; Zeng, X.; Zhuo, R. X.; Feng, J.; Zhang, X. Z. Biomaterials 2015, 52,281

[22] Hao, Y. W.; Zheng, C. X.; Wang, L.; Hu, Y. J.; Guo, H. C.; Song, Q. L.; Zhang H L.; Zhang, Z. Z.; Zhang, Y. J. Mater. Chem. B 2017, 5, 2133.

[23] Zhu, X. Y.; Wu, C. H.; Qiu, S. H.; Yuan, X. L.; Li, L. Nutr. Metab. 2017, 14, 60 .

[24] Xu, Y.; Wang, L. M.; He, J.; Bi, Y. F.; Li, M.; Wang, T. G.; Wang, L. H.; Jiang, Y.; Dai, M.; Lu, J. L.; Xu, M.; Li, Y. C.; Hu, N.; Li, J. H.; Mi, S. Q.; Chen, C. S.; Li, G. W.; Mu, Y. M.; Zhao, J. J.; Kong, L. Z.; Chen, J. L.; Lai, S. H.; Wang, W. Q.; Zhao, W. H.; Ning, G. $J$. Am. Med. Assoc. 2013, 310, 948 .

[25] Shah, R. B.; Patel, M.; Maahs, D. M.; Shah, V. N. Int. J. Pharm. Invest. 2016, 6, 1 .

[26] Wang, Y. X.; Chai, Z. H.; Ma, L. Y.; Shi, C. S.; Shen, T. F.; Song, J. RSC $A d v$. 2014, 4, 53877.

[27] Zhang, Y. H.; Zhang, Y. M.; Zhao, Q. H.; Liu, Y. Sci. Rep. 2016, 6, 22654.

[28] Gao, L.; Wang, T. T.; Jia, K. K.; Xuan Wu, X.; Yao, C. H.; Shao, W.; Zhang, D. M.; Hu, X. Y.; Wang, L. Y. Chem.-Eur. J. 2017, 23, 6605.

[29] Zuo, M. Z.; Qian, W. R.; Xu, Z. Q.; Shao, W.; Hu, X. Y.; Zhang, D. M.; Jiang, J. L.; Sun, X. Q.; Wang, L. Y. Small 2018, 14, 1801942.

[30] Wang, J. Q.; Yu, J.; Zhang, Y. Q.; Zhang, X. D.; Kahkoska, A. R.; Chen, G. J.; Wang, Z. J.; Sun, W. J.; Cai, L. L.; Chen, Z. W.; Qian, C. G.; Shen, Q. D.; Khademhosseini, A.; Buse, J. B.; Gu, Z. Sci. Adv. 2019, 5, eaaw4357.

[31] Yu, J. C.; Wang, J. Q.; Zhang, Y. Q.; Chen, G. J.; Mao, W. W.; Ye, Y. Q.; Kahkoska, A. R.; Buse, J. B.; Langer, R.; Gu, Z. Nat. Biomed. Eng. 2020, 4, 499.

[32] Akhtar, N.; Akram, M.; Asif, H. M.; Usmanghani, K.; Shah, S. M. A.; Rao, S. A.; Uzair, M.; Shaheen, G.; Ahmad, K. J. Med. Plants Res. 2011, 5, 1812

[33] Miyazawa, H.; Hirai, K.; Ookawara, S.; Ishibashi, K.; Morishita, Y. Nano Rev. Exp. 2017, 8, 1331099.

[34] Yang, B.; Jia, H. Z.; Wang, X. L.; Chen, S.; Zhang, X. Z.; Zhuo, R. X.; Feng, J. Adv. Healthcare Mater. 2014, 3, 596.

[35] Jeon, J. H.; Park, J. H.; Kim, T. J. Ind. Eng. Chem. 2019, 75, 148.

[36] Liu, X.; Xiang, J. J.; Zhu, D. C.; Jiang, L. M.; Zhou, Z. X.; Tang, J. B.; Liu, X. R.; Huang, Y. Z.; Shen, Y. Q. Adv. Mater. 2016, 28 , 1743.

[37] Elvira, C.; Gallardo, A.; San Roman, J.; Cifuentes, A. Molecules $\mathbf{2 0 0 5}, 10,114$. 
[38] Tan, J. P. K.; Voo, Z. X.; Lim, S.; Venkataraman, S.; Ng, K. M.; Gao, S. J.; Hedrick, J. L.; Yang, Y. Y. Nanomedicine 2019, 17, 236.

[39] Kim, J.; Lee, J.; Lee, Y. M.; Pramanick, S.; Im, S.; Kim, W. J. J. Controlled Release 2017, 259, 203.

[40] Zhang, Y. H.; Zhang, Y. M.; Yu, J.; Wang, J.; Liu, Y. Chem. Commun. 2019, 55, 1164.

[41] Chai, Y.; Xu, K.; Chang, H. B.; Zhang, P. Y. Chem. Res. 2018, 29, 522 (in Chinese). (柴云, 许凯, 常海波, 张普玉, 化学研究, 2018, 29, 522.)

[42] Li, Y. P.; Xiao, W. W.; Xiao, K.; Berti, L.; Luo, J. T.; Tseng, H. P.; Fung, G.; Lam, K. S. Angew. Chem., Int. Ed. 2012, 51, 2864.

[43] Han, Y.; Yin, W.; Li, J. J.; Zhao, H.; Zha, Z. S.; Ke, W. D.; Wang, Y. H.; He, C. X.; Ge, Z. S. J. Controlled Release 2018, 273, 30.

[44] Mehta, P.; Kadam, S.; Pawar, A.; Bothiraja, C. New J. Chem. 2019, 43,8396 .

[45] Jiang, W. F.; Zhou, Y. F.; Yan, D. Y. Chem. Soc. Rev. 2015, 44, 3874.

[46] Jia, H. Z.; Zhu, J. Y.; Wang, X. L.; Cheng, H.; Chen, G.; Zhao, Y. F.; Zeng, X.; Feng, J.; Zhang, X. Z.; Zhuo, R. X. Biomaterials 2014, 35, 5240.

[47] Long, Z.; Liu, M. Y.; Mao, L. C.; Zeng, G. J.; Wan, Q.; Xu, D. Z.; Deng, F. J.; Huang, H. Y.; Zhang, X. Y.; Wei, Y. Colloids Surf., B 2017, 150, 114.

[48] Liu, C. Y.; Shao, N. M.; Wang, Y. T.; Cheng, Y. Y. Adv. Healthcare Mater. 2016, 5, 584.

[49] Hu, Y. C.; Wang, Y. Z.; Wang, S. L. J. Shenyang Pharm. Univ.. 2010, 27, 961 (in Chinese). (胡延臣, 王彦竹, 王思玲，沈阳药科大学学报, 2010, 27, 961.)

[50] Regi, M. V.; Ramila, A.; del Real, R. P.; Pariente, J. P. Chem. Mater. 2001, 13, 308 .

[51] Wang, H. G.; Liu, X. H. Chin. J. Hosp. Pharm. 2019, 39, 1099 (in Chinese). (王海刚, 刘向红, 中国医院药学杂志, 2019, 39, 1099.)

[52] Hu, C. L.; Yu, L. X.; Zheng, Z.; Wang, J.; Liu, Y.; Jiang, Y. F.; Tong, G. Z.; Zhou, Y. J.; Wang, X. L. RSC Adv. 2015, 5, 85436.

[53] Tan, L.; Yang, M. Y.; Wu, H. U.; Tang, Z. W.; Xiao, J. Y.; Liu, C. J.; Zhuo, R. X. ACS Appl. Mater. Interfaces 2015, 7, 6310.

[54] Qiu, X. L.; Li, Q. L.; Zhou, Y.; Jin, X. Y.; Qi, A. D.; Yang, Y. W. Chem. Commun. 2015, 20, 4237

[55] Xu, Y. H.; Shi, W.; Li, H. Y.; Li, X. H.; Ma, H. M. ChemMedChem 2019, 14, 1079.

[56] Aguirre-Chagala, Y. E.; Santos, J. L.; Aguilar-Castillo, B. A.; Her-
rera-Alonso, M. ACS Macro Lett. 2014, 3, 353.

[57] Zhao, Z. K.; Zhang, Y.; Tian, C. L.; Yin, T. J.; Zhang, C. Biomater. Sci. 2018, 6, 2605 .

[58] Bai, J. A.; Tian, Y.; Liu, F. Z.; Li, X. L.; Shao, Y.; Lu, X. T.; Wang, J. T.; Zhu, G. Q.; Xue, B. Y.; Liu, M.; Hu, P.; He, N.; Tang, Q. Y. ACS Appl. Mater. Interfaces 2019, 11, 18111.

[59] Chen, Y. J.; Han, H. J.; Tong, H. X.; Chen, T. T.; Wang, H. B.; Ji, J.; Jin, Q. ACS Appl. Mater. Interfaces 2016, 8, 21185.

[60] Fan, B.; Kang, L.; Chen, L. P.; Sun, P.; Jin, M. J.; Wang, Q. M.; Bea, Y. H.; Huang, W.; Gao, Z. G. Theranostics 2017, 7, 357.

[61] Kim, J.; Lee, Y. M.; Kim, H.; Park, D.; Kim, J.; Kim, W. J. Biomaterials 2016, 75, 102.

[62] Wang, C. Y.; Qi, P. L.; Lu, Y.; Liu, L.; Zhang, Y. N.; Sheng, Q. L; Wang, T. S.; Zhang, M. Y.; Wang, R.; Song, S. Y. Drug Delivery 2020, 27, 344

[63] Zhang, P; Xu, Q. N.; Li, X. F.; Wang, Y. X. Mater. Sci. Eng., C 2020, 108, 110396.

[64] Chen, W. Z.; Zhen, X.; Wu, W.; Jiang, X. Q. Sci. China: Chem. 2020, 63, 648 .

[65] Mansour, O.; El Joukhar, I.; Belbekhouche, S. React. Funct. Polym. 2019, 145, 104377 .

[66] Szatrowski, T. P.; Nathan, C. F. Cancer Res. 1991, 51, 794.

[67] Li, C. T.; Pan, R. J.; Li, P. Y.; Guan, Q. H.; Ao, J. P.; Wang, K.; Xu, L.; Liang, X. F.; Jin, X.; Zhang, C.; Zhu, X. Y. Anal. Chem. 2017, 89, 5967.

[68] Zhang, Q. X.; Zhang, F. Z.; Chen, Y.; Dou, Y.; Tao, H.; Zhang, D. L.; Wang, R. B.; Li, X. H.; Zhang, J. X. Chem. Mater. 2017, 29, 8221.

[69] Antunes, F.; Cadenas, E. Free Radicals Biol. Med. 2001, 30, 1008.

[70] Ye, M. Z.; Han, Y. X.; Tang, J. B.; Piao, Y.; Liu, X. R.; Zhou, Z. X.; Gao, J. Q.; Rao, J. H.; Shen, Y. Q. Adv. Mater. 2017, 29, 1702342.

[71] Yin, W.; Li, J. J.; Ke, W. D.; Zha, Z. S.; Ge, Z. S. ACS Appl. Mater. Interfaces 2017, 9, 29538.

[72] Zhou, J.; Yu, G. C.; Yang, J.; Shi, B. B.; Ye, B. Y.; Wang, M. B.; Huang, F. H.; Stang, P. J. Chem. Mater. 2020, 32, 4564.

[73] Qie, S. Y.; Hao, Y.; Liu, Z. J.; Wang, J.; Xi, J. N. Acta Chim. Sinica 2020, 78, 232 (in Chinese) (郄淑燕，郝荣，刘宗建，王锦，席家宁，化学学报，2020，78， 232.)

[74] Zhou, J. Y.; Tang, Q. J.; Zhong, J. X.; Lei, Z. T.; Luo, H. P.; Tong, Z. Z.; Jang, G. H.; Liu, X. D. J. Mater. Sci. 2018, 53, 14063.

(Cheng, F.) 